Full length article

\title{
Mutual influence of gold and silver nanoparticles on Tris-(2,2'bipyridine)-Ru(II) core complexes: Post-functionalization processes, optical and electrochemical investigations
}

\author{
Frédéric Dumur ${ }^{\mathrm{a},}$, , Audrey Guerlin ${ }^{\mathrm{f}}$, Anaïs Lehoux ${ }^{\mathrm{c}}$, P.R. Selvakannan ${ }^{\mathrm{g}}$, Fabien Miomandre ${ }^{\mathrm{d}}$, \\ Rachel Méallet-Renault ${ }^{\text {h, Mateusz Rebarz }}{ }^{\mathrm{i}}$, Michel Sliwa ${ }^{\mathrm{i}}$, Eddy Dumas ${ }^{\mathrm{c}}$, Loïc Le Pleux ${ }^{\mathrm{e}}$, Yann Pellegrin ${ }^{\mathrm{e}}$, \\ Fabrice Odobel ${ }^{\mathrm{e}}$, Cédric R. Mayer ${ }^{\mathrm{b}}$ * \\ ${ }^{a}$ Aix Marseille Univ, CNRS, ICR, UMR 7273, F-13397 Marseille, France \\ b Département de Chimie, UFR des Sciences, Université de Versailles Saint-Quentin-en-Yvelines, 78035 Versailles, France \\ ${ }^{\mathrm{c}}$ Institut Lavoisier de Versailles, UMR-CNRS 8180, Université de Versailles Saint-Quentin-en-Yvelines, 78035 Versailles, France \\ d Laboratoire de Photophysique et Photochimie Supramoléculaires et Macromoléculaires, UMR CNRS 8531, ENS Paris-Saclay, 94235 Cachan, France \\ e Chimie et Interdisciplinarité: Synthèse, Analyse, Modélisation (CEISAM), UMR-CNRS 6230, Université de Nantes, 44322 Nantes, France \\ ${ }^{\mathrm{f}}$ Université Paris Diderot, UFR de Chimie, 15 Rue Jean Antoine de Baïf, 75013 Paris, France \\ ${ }^{g}$ Research Fellow School of Science, RMIT University, Melbourne, 3001, VIC, Australia \\ ${ }^{\mathrm{h}}$ Institut des Sciences Moléculaires d'Orsay (ISMO) - UMR 8214, Université Paris-Saclay, Université Paris-Sud, 91405 Orsay Cedex, France \\ ${ }^{\mathrm{i}}$ Univ. Lille, CNRS, UMR 8516 - LASIR - Laboratoire de Spectrochimie Infrarouge et Raman, F-59000 Lille, France
}

\section{A R T I C L E I N F O}

Keywords:

Polypyridyl ruthenium complexes

$\mathrm{Ag}$

$\mathrm{Au}$

Nanoparticles

Spectroscopies

Electrochemistry

\begin{abstract}
A B S T R A C T
The synthesis, reactivity and properties of a series of four polypyridyl ruthenium complexes have been studied. These complexes were used to post-functionalize preformed $3 \mathrm{~nm}$ silver and gold nanoparticles (NPs) in water and in dichloromethane (DCM). We studied the influence of the grafted complexes on the formation process and stability of the colloidal solutions and we investigated the optical and electrochemical properties of the final nanocomposites. Among the series of four ruthenium complexes, three novel heteroleptic complexes (1-3) bearing one pyridine, one amine or two carboxydithioic acid pendant groups were synthesized and reacted with preformed Au-NPs and Ag-NPs. Results were compared to those obtained with the model $\left[\mathrm{Ru}(\mathrm{bpy})_{3}\right]^{2+}$ complex (4). The strength of the interaction between the anchoring group and the surface of NPs influenced the size, shape and stability of the final nanocomposites. Polar solvent such as water induced aggregation and lead to unstable nanocomposites. Stationary and time resolved luminescence of grafted nanocomposites (1-3) showed that the luminescence of complexes were completely quenched (lifetime and emission quantum yield) in water by electron transfer processes, moreover electrical measurements rationalize that Ag nanocomposites exhibit the stronger quenching due to a lower oxidation potential. It also showed a current enhancement associated with double layer charging of the metal nanoparticle cores.
\end{abstract}

\section{Introduction}

Over the past three decades, tris-(2,2'-bipyridine)ruthenium(II) has been one of the most studied coordination complex in photochemistry [1]. The reasons are numerous but its chemical and electrochemical robustness [2], the facile design and the modularity of the ligands [3] and its long-lived emission motivated the synthesis of various derivatives [4]. The use of Ru(II) polypyridine complexes is broad and covers a wide range of properties and applications such as optoelectronics de- vices [5], nonlinear optics [6], bio-imaging [7], bio-analysis [8], catalysis [9], solar cells [10] and nanosciences [11]. Even if considerable efforts have been devoted to the design of new molecular derivatives for the above mentioned applications, molecular building blocks (MBBs) have also received a great deal of interest for material sciences. This interest is motivated by the unique properties brought by the Ru-MBB to the host matrixes and the multifunctional character of the resulting materials. They have been incorporated in liquid crystals [12], hybrid polymers (metallopolymers) [13], dendrimers (metallodendrimers)

\footnotetext{
* Corresponding authors.

Email address: frederic.dumur@univ-amu.fr (F. Dumur)
} 
[14], micelles [15], sol-gel materials [16], silica nanomaterials [17] and more recently grafted onto oxometallic [18] and metallic nanoparticles [19]. Parallel to Ru(II) coordination complexes, silver and gold nanoparticles (NPs) constitute another active field of research. Both types of NPs are of particular interest in nanosciences and they know an ever increasing research effort due to their intrinsic physicochemical properties (in particular electronic and optical properties) [20] and their facile functionalization [21]. Domains of applications are ranging from catalysis [22], opto-electronics [23] to biomedical materials [24]. The grafting of redox active and/or fluorophore probes onto metallic nanoparticles has recently drawn an intense research interest [25]. Electrochemical studies realized by Murray's group clearly demonstrated the role of electron reservoir played by the nanoparticles and the possibility to modulate the charge states of the NPs by means of the applied potential [26]. The ability to confine and control the number of redox/fluorophore centres on the NPs also leads to characteristic electrochemical behaviours showing a concerted redox process combined with a fluorophore emission quenching [27]. In all these reports, the redox active centres are not electronically connected to the NPs and these systems are comparable to functionalized dendrimers, the NPs simply acting as linkers [28]. Polypyridyl Ru(II) complexes are excellent redox and luminescent probes. Consequently, the grafting of these molecular units onto the surface of nano-objects with an effective control of the communication between both components of the nanocomposites is of great interest for fundamental research but also for potential applications. Studies of the interactions between polypyridyl ruthenium complexes and metallic nanoparticles appeared only recently in the literature. Most of these ruthenium tris-bipyridine complexes are either interacting with the NPs via electrostatic interactions [29], or they are grafted onto the NPs via long aliphatic chains functionalized by various anchoring end-groups such as thiols [30], dithiocarbamate [31], or aromatic triamine [32]. However, both strategies are not suitable for efficient electronic transfers due to the absence of conjugation between both partners.

In the past five years, our group has been involved in the synthesis of gold and silver NPs stabilized and functionalized by ruthenium complexes via rigid and fully conjugated bridges [33]. The physicochemical properties of these nanocomposites and more specifically their electrochemical and luminescence properties were first discussed [34]. To date, few works have thoroughly been devoted to the study of the alteration of the luminescence of inorganic fluorophores when grafted onto metallic nanoparticles. The quenching of luminescence is often observed because different deactivation pathways, inter/intramolecular
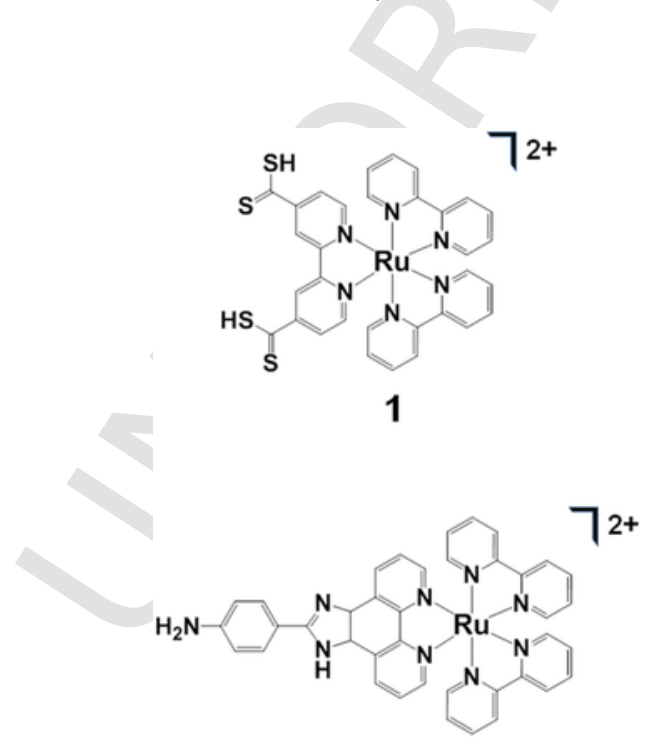

3 interaction, energy and/or electron transfer are possible between the fluorophore and the NP. In both cases, these processes are sensitive to the nature of the bridge (length, conjugation, rigidity ...) between the fluorophore and the NP and are also sensitive to the size and the shape of the NP. Different approaches are possible to functionalize Ag-NPs or Au-NPs but the most common method remains the place exchange of the stabilizing agent, initially required to generate the NPs, by the desired functionalizing agent [35]. The place exchange approach allows for a better control of the doping amount of functionalizing agent on the NPs and is applicable in water and organic solvents such as chloroform or toluene. In these media, the initial stabilizing agent (sodium citrate in water and long alkylamine or alkylcarboxylic acid chains in organic solvent) is easily substitutable by the functionalizing agent.

Herein, we investigated the post-functionalization of preformed Ag-NPs and Au-NPs by tris-(2,2'-bipyridine)Ru(II) derivatives and the reciprocal influence between the NPs and the ruthenium complexes on the stability and on the physicochemical properties of the final nanocomposites. Au-NPs and Ag-NPs were used to investigate the influence of oxidation potential and of the plasmon band energy (lower and higher energy respectively) compare to the one of the MLCT band of tris-(2,2'-bipyridine)Ru(II) derivatives. Four complexes have been used in this work, namely [(bpy) ${ }_{2} \mathrm{Ru}\left(4,4^{\prime}\right.$-(carboxydithioic acid)-2,2'-bipyridine $)(\mathrm{X})_{2} \quad\left(1 . \mathrm{X}_{2}\right), \quad\left[(\mathrm{bpy})_{2} \mathrm{Ru}((4-(4-p y r i d i n y l) p h e n y l) i m i-\right.$ dazo[4,5-f] $[1,10]$ phenanthroline $)](\mathrm{X})_{2}$ [(bpy) ${ }_{2} \mathrm{Ru}((4$-aminophenyl)imidazo[4,5- $f][1,10]$ phenanthroline $\left.)\right](\mathrm{X})_{2}$ $\left(3 . \mathrm{X}_{2}\right)$ and $\left[(\mathrm{bpy})_{3} \mathrm{Ru}\right](\mathrm{X})_{2}\left(4 . \mathrm{X}_{2}\right), \mathrm{X}^{-}=\mathrm{PF}_{6}{ }^{-}$or $\mathrm{Cl}^{-}$, in which we varied the nature of the anchoring group as well as the distance between the complex and the NP (Scheme 1). Complex 4 was prepared as a model to control the role of the anchoring groups (pyridine, amine and carboxydithioic acid) on the post-functionalization process. The mutual influence between metal-NPs and ruthenium complexes has been analyzed in two solvents, water and dichloromethane (DCM). The choice of both solvents was motivated by the fact that redox and luminescence properties of the resulting nanocomposites can be investigated in both organic and aqueous media simply by counteranion exchange. This influence has been investigated by TEM, UV/Vis, luminescence, time resolved spectroscopies and electrochemistry.

\section{Results and discussion}

\subsection{Synthesis of the proligands and complexes}

Four complexes have been used in this study (Scheme 1). The first one, $\mathbf{1}$, is structurally closely related to the well-known $\left[\mathrm{Ru}(\mathrm{bpy})_{3}\right]^{2+}$
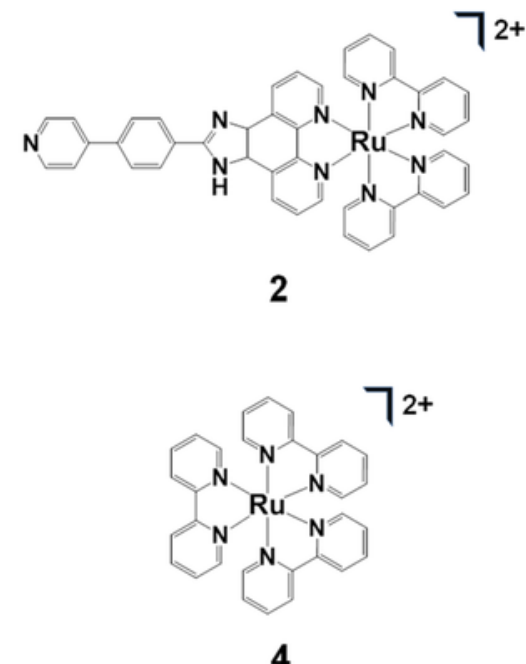

Scheme 1. Structures of the four complexes used in this study. 
(4) except that one bipyridine was functionalized by two carboxydithioic acid groups. The choice of carboxydithioic acid as anchoring group was motivated by its strong affinity for soft metals [31,36,37], which should ensure strong bonding to metal surfaces [38]. The preparation of $\mathbf{1}$ is relatively straightforward and consists of the oxidation of 4,4'-bis(chloromethyl)-2,2'-bipyridine directly on 5 [39] by elemental sulphur in the presence of sodium methanolate (Scheme 2) [40].

Complexes $\mathbf{2}$ and $\mathbf{3}$ were functionalized with phenylpyridine for $\mathbf{2}$ and aniline for 3 , since these groups proved to be effective anchoring groups to stabilize and to bind to metal NPs [33,41,42]. Conventional aliphatic thiols have not been used in this work to link the ruthenium complexes to the metal NPs because this type of binding has already been abundantly reported by Kamat et al. [43] and we wanted to favor electronic interactions by maintaining a conjugated linkage between both partners. Complex 2 was synthesized by reaction of $\left[(\text { bpy })_{2} \mathrm{Ru}\right.$ (phendione) $\mathrm{Cl}_{2}\left(6 . \mathrm{Cl}_{2}\right.$ ) with 4-(4-pyridinyl)benzaldehyde. Complexes 1, 2, 3 and 4 were isolated as hexafluorophosphate or chloride salts depending on the solvent further used to generate the final nanocomposites.

\subsection{Synthesis of the nanocomposites}

As already mentioned, the conventional method to functionalize Au-NP or Ag-NPs consists in the post-functionalization of preformed NPs. This method was also selected here. In previous works reported by our group, other methods have been described to functionalize metal NPs with metal complexes: the direct functionalization [33a], the post-functionalization by phase transfer [33c] or the generation of the metal complexes directly on NPs pre-functionalized with the appro- priate proligands [33b]. In the first two cases, the colloidal solutions contained a significant excess of free complexes which renders the luminescence investigations difficult. This problem is avoided with the third method but requires proligands able to stabilize the NPs before complexation and this strategy is not applicable to a large variety of complexes. These considerations prompted us to develop the post-functionalization of NPs by place exchange reaction (Scheme 3). Besides, this approach provides a better control of the size of the NPs and a better control of the amount of metal complexes grafted on the NPs. The place exchange process was performed in water and in DCM but can be extended to other solvents such as chloroform or toluene. The choice of both solvents is supported by the potential applications of the nanocomposites studied in this work. Indeed, Ru(II) complexes and metal NPs have potential applications in biology and optoelectronics. In the context of our work, one of the major difficulties was to obtain homogeneous colloidal solutions with uniform NPs of narrow size distribution and morphology stabilized with easily exchangeable surfactants. In aqueous solution, sodium citrate was used as initial surfactant in diluted medium. Ag-NPs and Au-NPs were obtained with a size distribution centred around $2.5 \mathrm{~nm}$. In DCM, 1-hexadecylamine (HDA) or a mixture of HDA and oleic acid (OA) were used to prepare Au-NPs and Ag-NPs, respectively. In the case of Ag-NPs, OA was essential to obtain stable and homogeneous colloidal solutions. The mean size distribution of Au-NPs and Ag-NPs was determined to be $3 \pm 1 \mathrm{~nm}$. The post-functionalization was performed by mixing the preformed metal NPs with the appropriate ruthenium complex. The amount of ruthenium complexes added in each colloidal solution was chosen following this calculation: we first estimated the ratio between the surface of one NP and the projected surface of each complex onto a plane surface. Then, for all colloidal solutions, the amount of complexes introduced

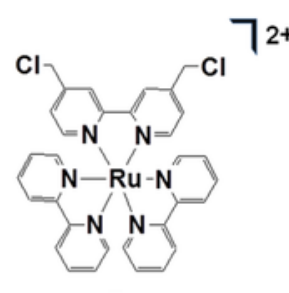

5

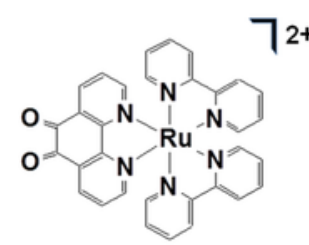

6
$-1-\mathrm{S}_{8} / \mathrm{MeONa}$

$\mathrm{MeOH}-\mathrm{RT}$

-2- aqueous $\mathrm{KPF}_{6}$

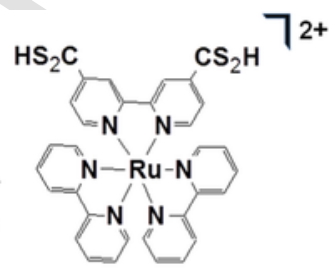

1
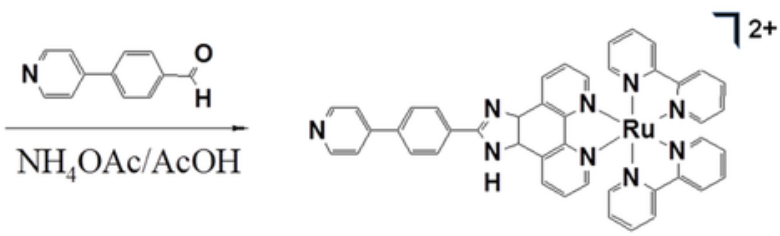

2

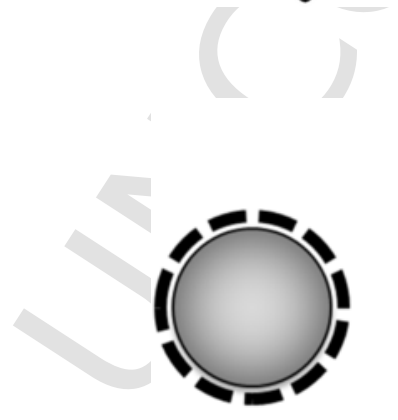

Scheme 2. Synthesis of 1 and 2.
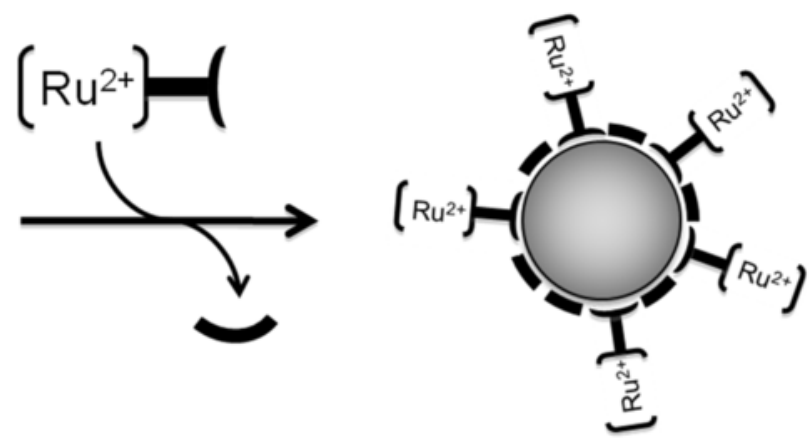

Scheme 3. Post-functionalization process used in this study. 
was about $20 \%$ of the theoretical amount required for the maximal coverage. Except for the electrochemistry measurements, we voluntarily added a low concentration of ruthenium complexes in order to limit the communication between them once grafted onto the surface of the NPs because it was already observed by Kamat et al. that electron transfer between different $\mathrm{Ru}(\mathrm{bpy})_{3}{ }^{2+}$ could be observed [43].

\subsection{NPs in water}

In aqueous solution, the nature of the metal NPs had a strong influence on the stability of the final nanocomposites. Noticeable ligand effects were also evidenced with a strong impact on the form, the size and the stability of the functionalized NPs. Immediately after addition of the ruthenium complexes, and depending on the anchoring group, the colour of the gold colloidal solutions changed, more or less rapidly, from red to purple and finally blue. The colour of the solutions is highly indicative of the stability of the colloidal solutions. Without anchoring group, as in the case of complex 4, aggregates lead to a blue solution and a precipitate was rapidly observed. The stability of the colloidal solutions increased with the strength of the interaction between the anchoring group and the surface of the Au-NP in the following order: amine (3), pyridine (2) and carboxydithioic acid (1) (see Fig. S1). However, even with strong anchoring groups such as phenylpyridine or carboxydithioic acid, Au-NPs flocculated after one night. The instability of the gold colloidal solution can be explained by these mechanisms: in the case of complexes $\mathbf{1}$ and $\mathbf{2}$, these ruthenium complexes first replace citrate anions onto the surface of Au-NPs. Then, in a second step, the positive charges brought by the complexes are neutralized by citrates anions, remaining in solution, leading to the progressive aggregation of Au-NPs (Scheme 4a). This type of neutralization, due to the presence of citrates anions, was recently described with other cationic molecules [44]. The aggregation phenomenon was confirmed by TEM, as shown in Fig. 1 for complex 2. For complex 3, for which a weaker interaction is expected between the anchoring group and the surface of the Au-NP, and for complex 4 an even faster aggregation was observed compared to complexes 1 and 2 . In the case of cationic complex 4, electrostatic interactions with citrate anions surrounding the Au-NPs are responsible for the aggregation of the Au-NPs (Scheme 4a).
Concerning the functionalization of Ag-NPs in water, a specific strategy was developed due to the difference of reactivity between Au-NPs and Ag-NPs. This difference originates from the surface charge of the NPs: Ag-NPs are positively charged while Au-NPs are negatively charged [45]. Due to this major difference, addition of 4 , for example, did not immediately induce the aggregation of Ag-NPs and the colloidal solution was stable for several days (Fig. S1b). The influence of the anchoring group on the size of the final NPs is more pronounced with Ag-NPs compared to Au-NPs (Scheme 4b). For example, after addition of complex 4, the solution remained stable without modification of the colour. For all the other complexes, the colour of the solutions turned to brown yellow for $\mathbf{3}$, to red for $\mathbf{2}$ and dark red for $\mathbf{1}$. The stability of these solutions was maintained for a few days before aggregation. Interpretation of these different colors, depending on the nature of the anchoring group, has been given using DLS, TEM and UV/Vis spectroscopy. DLS clearly showed a modification of the size of the silver nanocomposites after addition of the ruthenium complexes. This modification was even more significant when the anchoring group had a higher affinity for the metallic surface (Fig. 2). $1 \mathrm{~h}$ after addition of 4 , no evolution of the size of the nanocomposites was observed (Fig. 2). For the other complexes, two populations of nanocomposites were detected; one with an average hydrodynamic diameter $\left(d_{\mathrm{h}}\right)$ centered at 2-3 nm for all three complexes and a second one with $d_{\mathrm{h}}$ varying with the nature of the anchoring group. For complex 3 , the $d_{\mathrm{h}}$ of the second population was centered around $20 \mathrm{~nm}$, and for complexes 2 and 1 , the size distribution was broader and centered around 35 and $50 \mathrm{~nm}$, respectively. For complexes 1, 2 and 3, DLS analysis shows that the evolution of the size of the silver nanocomposites follows a two steps process (Figs. S2 and S3 and Scheme 4b). In the first step, two populations of nanocomposites were observed, after five minutes of stirring, with average $d_{\mathrm{h}}$ values centered around 7 and $20 \mathrm{~nm}$ for 1 and around 4 and $9 \mathrm{~nm}$ for 2 . During the second step, the systems evolved towards the two final populations observed with the largest nanocomposites clearly growing at the expense of the smallest ones (Ostwald ripening process). The final composition of the colloidal suspensions was confirmed by TEM analysis (one drop of the solution studied by DLS $1 \mathrm{~h}$ after addition of the complexes was taken and dried on a copper grid). TEM pictures (Figs. 3 and S3) showed Ag-NPs with a size distribution in accordance with the results obtained by DLS. Small Ag-NPs, with a

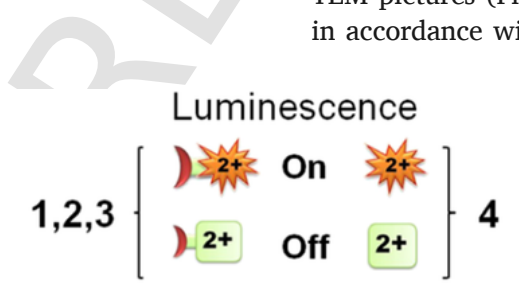

a)

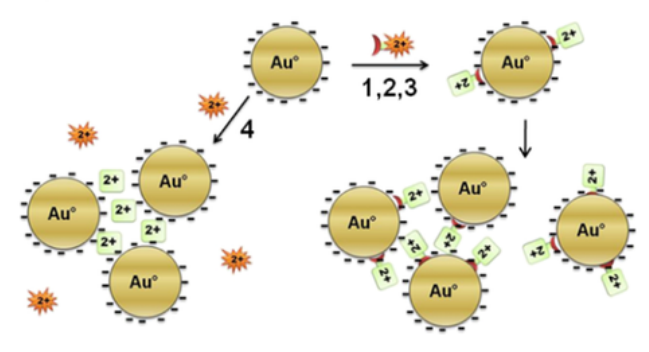

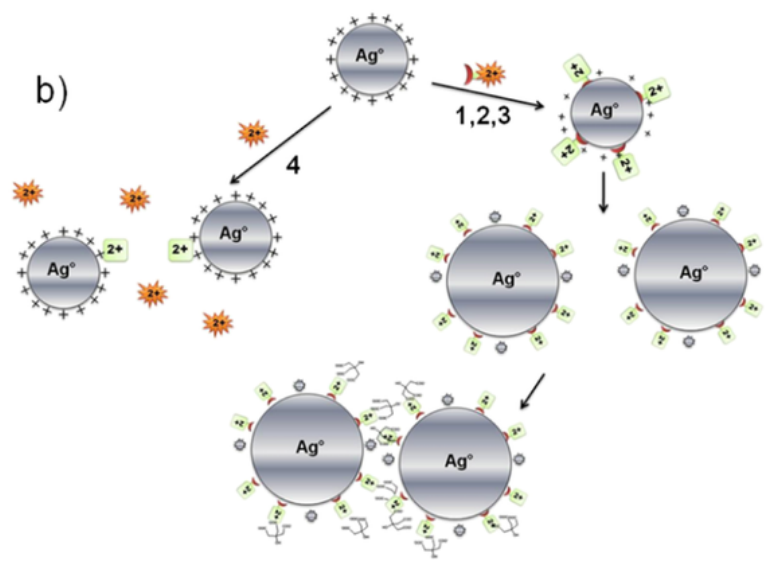

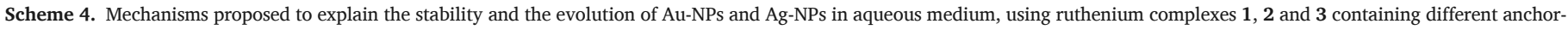
ing groups and 4. 


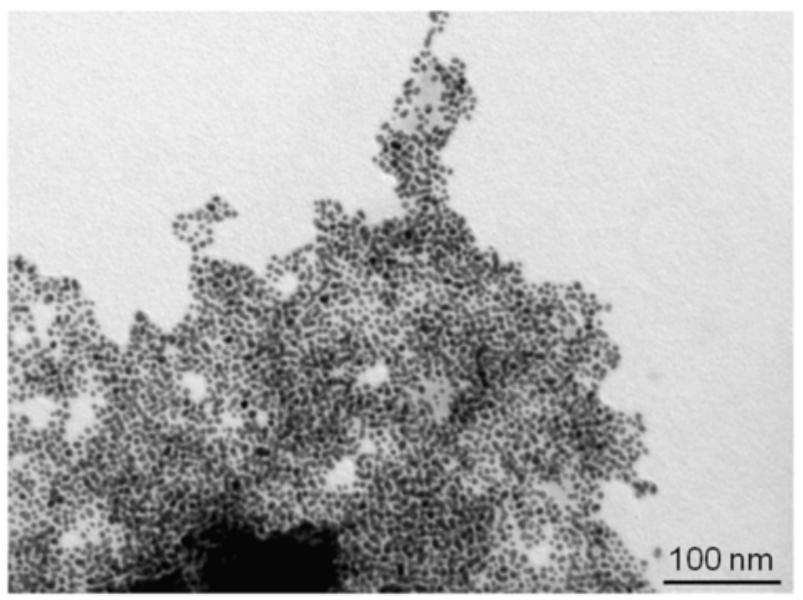

Fig. 1. TEM image of Au-NPs in water $1 \mathrm{~h}$ after addition of 2.

mean diameter of about $2 \mathrm{~nm}$, surround bigger Ag-NPs with a mean diameter varying with the nature of the anchoring group. The size of the bigger particle follows the strength of the interaction between the anchoring group and the surface of nanoparticles. The post-functionalization of Ag-NPs in aqueous medium with ruthenium complexes clearly induced a modification of the preformed Ag-NPs leading to two distinct populations in size, following an Ostwald ripening process. The extent of this phenomenon is obviously correlated with the nature of the anchoring group and therefore with the strength of the interaction between the ruthenium complex and the silver surface. Kamat group observed by TEM surface modification of gold nanorods after post-functionalization with $\mathrm{Ru}(\mathrm{bpy})_{3}-\mathrm{C}_{5}-\mathrm{SH}$ which lead to some aggregation. This was explained as an induced surface corrosion from the thiol due to the redox nature of the $\mathrm{Ru}(\mathrm{bpy})_{3}{ }^{2+}$ [46]. Similar hypothesis could be also involved in our case because the aggregation process (size of nanoparticles) increases with the strength of the interaction between the anchoring groups. Finally, it has to be noticed that, as for Au-NPs, Ag-NPs precipitated after several days. The redox corrosion is stronger in our case because no aliphatic bond was used between $\mathrm{Ru}(\mathrm{bpy})_{3}{ }^{2+}$ and NPs and it could explained that the NPs are not stable in water after several days in comparison to DCM, electron transfer and redox corrosion are facilitated in water. This precipitation can also been attributed to the presence of citrate anions screening the repulsive positive charges brought by the ruthenium complexes located at the surface of the Ag-NPs and responsible for the temporary stability of the colloidal solutions (Scheme 4b).

\subsection{NPs in DCM}

In DCM, the final colloidal solution showed a greater stability compared with the corresponding aqueous solutions and this can be explained by the absence of aggregation due to corrosion process and also to electrostatic interactions between charged species remaining in solution and the charged NPs. All solutions were stirred for $48 \mathrm{~h}$ before analysis. TEM images evidenced significant differences of reactivity depending on the nature of the metal NP and also depending on the nature of the ruthenium complex. TEM images of preformed Au-NPs, stabilized with HDA, showed spatially well-ordered Au-NPs due to interdigitation of the long alkyl chains of HDA surrounding nearby NPs [47]. In the same conditions, preformed Au-NPs reacted with 4 did not induced significant modification of the size of the Au-NPs or even of the organization of the Au-NPs on the grid. In contrast, reaction of the preformed Au-NPs with the other ruthenium complexes induced a noticeable alteration of the spatial organization of the Au-NPs explained by the partial replacement of HDA by ruthenium complexes onto the surface of the Au-NPs. The stronger was the interaction between the anchoring group of the ruthenium complexes and the surface of the Au-NPs, the more efficient was the substitution of HDA by ruthenium complexes (Fig. 4a). Indeed, this substitution is more effective in the order $\mathbf{1}>\mathbf{2}>\mathbf{3}$. It has to be noticed that for all complexes, no noticeable modification of the size of the Au-NPs was observed after post-functionalization. This implied that solvent polarity is playing a big role in the stability and the absence or existence of aggregation process. As it was mentioned previously the corrosion process due to the redox nature of $\mathrm{Ru}(\mathrm{bpy})_{3}{ }^{2+}$ through the anchoring group is happening more strongly in polar solvent. In the case of Ag-NPs, the initial spatial organization of the preformed Ag-NPs is no longer observed by TEM with 1,2 and 3 . While the post-functionalization process using 1 and $\mathbf{3}$ has only a minor effect on the size and shape of the final nanocomposites, reaction of $\mathbf{2}$ on preformed Ag-NPs drastically modified both the size and the shape of the latter (Fig. 4b). Two populations of Ag-NPs were observed, one similar to the preformed Ag-NPs and a second population of much bigger size, centered around $20 \mathrm{~nm}$, with facetted Ag-NPs (truncated nanotriangles, nanohexagons, nanorods ...). The reason why only complex $\mathbf{2}$ induced such a drastic modification of the size and shape of the preformed Ag-NPs remains yet unexplained.

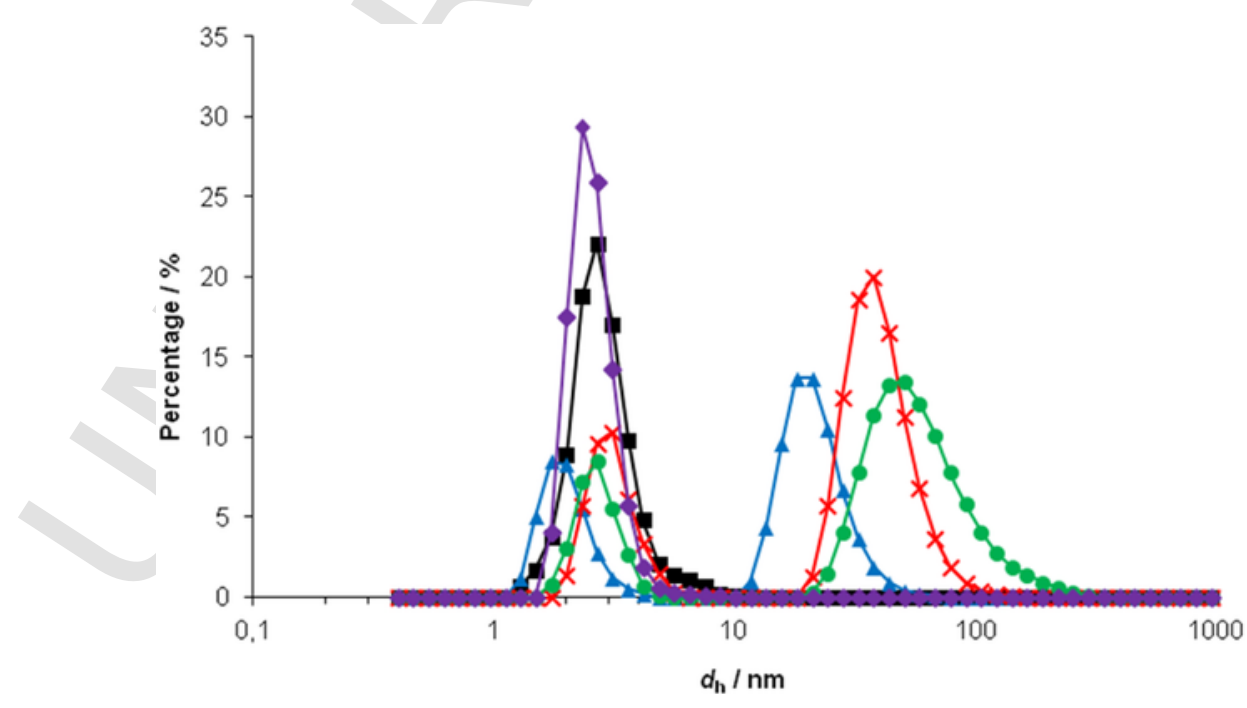

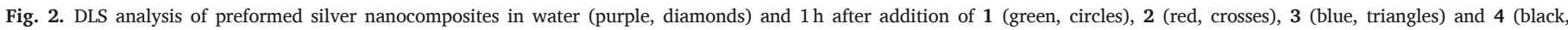
squares). (For interpretation of the references to colour in this figure legend, the reader is referred to the web version of this article.) 


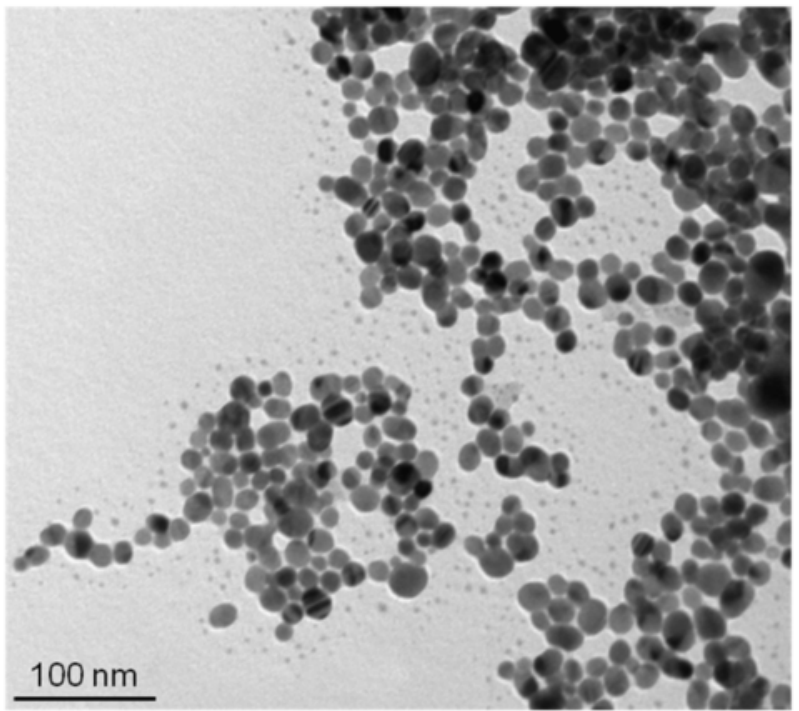

Fig. 3. TEM images of Ag-NPs in water $1 \mathrm{~h}$ after addition of $\mathbf{1}$.

However, in both solvents, water and DCM, it is obvious that the nature of the ruthenium complex, and more precisely the strength and the nature of the anchoring point of the complex, has much more influence on the size and shape of the final nanocomposites in the case of Ag-NPs than in the case of Au-NPs.

\subsection{UV/Vis stationary and time resolved spectroscopy}

Absorption spectra were recorded in two solvents, water and DCM, at room temperature using chloride or hexafluorophosphate salts of the ruthenium complexes. The most relevant spectroscopic results are summarized in Table 1.

Electronic absorption spectra of 1-4 in water and in DCM (Fig. 5a and d, respectively and see also Supporting Information) show intense bands in the UV region of the spectrum $(250-310 \mathrm{~nm})$. These bands can be attributed to $\pi \rightarrow \pi^{*}$ intraligand transitions from the bipyridine groups. In the $310-400 \mathrm{~nm}$ region, spectra of 2 and 3 present a feature band around $350 \mathrm{~nm}$ attributed to $\pi \rightarrow \pi^{*}$ intraligand transitions involving the imidazo(4,5-f)-(1,10)phenanthroline (PhenIm) moiety [48]. In the $400-600 \mathrm{~nm}$ region, ruthenium complexes exhibit a less intense and broader $\mathrm{d}\left(\mathrm{Ru}^{\mathrm{II}}\right) \rightarrow \pi^{*}(\mathrm{~L})$ MLCT band, with L corresponding to bipyridine and PhenIm [49]. As previously observed this MLCT band is slightly red-shifted compared to the reference compound 4 [50].

In aqueous solution, $\mathrm{UV}$-visible spectra of $\mathrm{Au}^{\circ}$ and $\mathrm{Ag}^{\circ}$ nanocomposites are in agreement with results obtained by DLS and TEM analyses (Fig. 5b and c). The amount of complexes added corresponded to one fifth of the calculated theoretical maximum covering. The starting gold colloidal solutions exhibited a surface plasmon band (SPB) at $509 \mathrm{~nm}$ in agreement with the size of the Au-NPs determined by TEM $[41,51]$. After addition of the different ruthenium complexes, the MLCT band was not discernible in the absorption spectra and only the $\pi \rightarrow \pi^{*}$ intraligand transitions were observed in the $230-300 \mathrm{~nm}$ region. Concerning the SPB, addition of 4 induced an important broadening of the band. This diffuse band seems to result from the contribution of two bands, centred around 540 and $655 \mathrm{~nm}$. This observation is in agreement with the rapid aggregation observed after addition of 4 . Addition of $\mathbf{3}$ and $\mathbf{2}$ caused a broadening and a red shift of the SPB (556 and $548 \mathrm{~nm}$, respectively) while the broadening and the red shift of the SPB is less important after addition of $1(527 \mathrm{~nm})$. These results are in agreement with the previous observations on the stability of the different colloidal solutions. After $1 \mathrm{~h}$, all solutions exhibited the same diffuse SPB illustrating the irreversible aggregation of the nanoparticles. Concerning Ag-NPs (Fig. 5c), the starting Ag-NPs exhibited a SPB at $395 \mathrm{~nm}$. After addition of 4 , the SPB was only slightly shifted. This result is in agreement with the previous observations, meaning that no interaction occurs between the cationic ruthenium complexes and the Ag-NPs. After addition of 3, the SPB was slightly shifted to $390 \mathrm{~nm}$ but the most striking feature was the appearance of a second SPB centred at $511 \mathrm{~nm}$. The influence of the addition of $\mathbf{2}$ and $\mathbf{1}$ was even more pronounced with the presence of the second SPB observed at 573 and $583 \mathrm{~nm}$, respectively. The small differences noticed in the spectra of $\mathbf{2}$ and $\mathbf{1}$ are in agreement with TEM and DLS observations, confirming in both cases the progressive growth of bigger Ag-NPs coexisting with smaller ones and the formation of aggregates in solution (Fig. S4).

Absorption spectra in DCM are gathered in Fig. 5d-f. With Au-NPs and with Ag-NPs the presence of the ruthenium complexes was only asserted by the $\pi \rightarrow \pi^{*}$ transition in the $235-300 \mathrm{~nm}$ region. Concerning Au-NPs (Fig. 5e), the position of the SPB was only slightly modified after addition of the ruthenium complexes, with a maximum red shift of $12 \mathrm{~nm}$ for 2. These observations are in full agreement with the TEM analyses showing the absence of noticeable modification of the size of the Au-NPs after post-functionalization. In the case of Ag-NPs, the post- a)
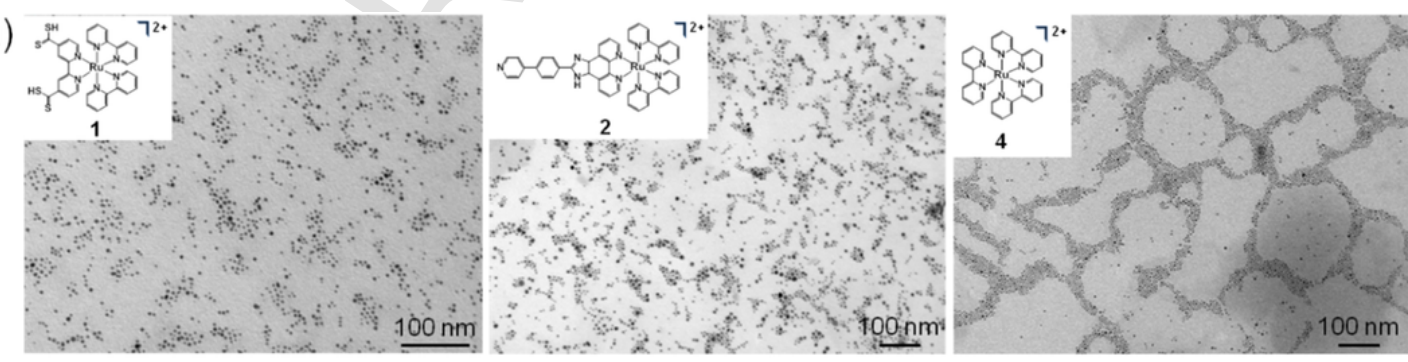

b)
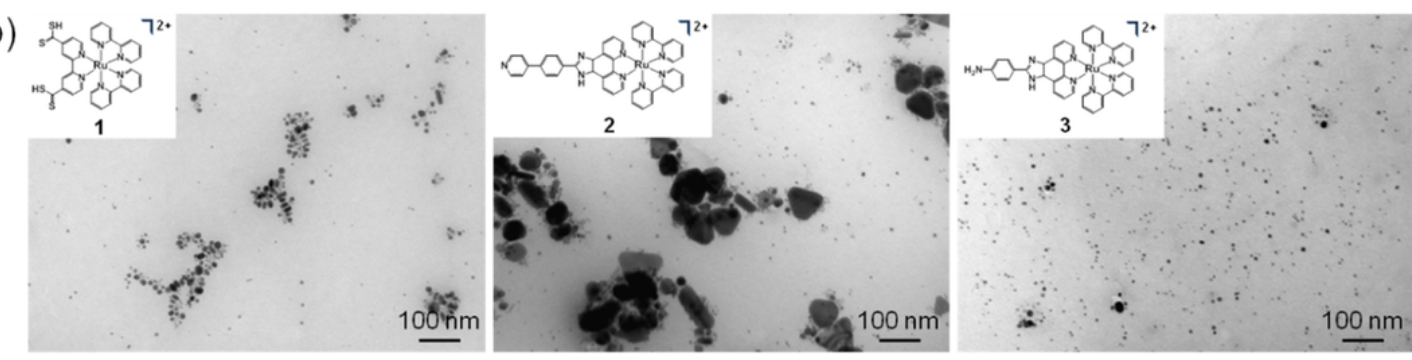

Fig. 4. TEM images in DCM of a) Au-NPs and b) Ag-NPs, $48 \mathrm{~h}$ after addition of the ruthenium complexes (see inserted scheme). 
Table 1

Electronic absorption data and assignment.

\begin{tabular}{|c|c|c|c|c|c|c|}
\hline Complexes $^{\mathrm{a}}$ & $\lambda_{\max }[\mathrm{nm}]$ & $\varepsilon\left[\mathrm{M}^{-1} \mathrm{~cm}^{-1}\right] \pm 15 \%$ & Assignment & Solvent & Metal & $\lambda_{\max } \mathrm{SPB}[\mathrm{nm}]$ \\
\hline & & & & $\mathrm{CH}_{2} \mathrm{Cl}_{2}$ & Ag & 431 \\
\hline & & & & $\mathrm{CH}_{2} \mathrm{Cl}_{2}$ & $\mathrm{Au}$ & 509 \\
\hline & & & & $\mathrm{H}_{2} \mathrm{O}$ & Ag & 395 \\
\hline & & & & $\mathrm{H}_{2} \mathrm{O}$ & $\mathrm{Au}$ & 509 \\
\hline \multirow[t]{4}{*}{1} & 457 & 13,140 & $d \rightarrow \pi^{*}$ & $\mathrm{CH}_{2} \mathrm{Cl}_{2}$ & Ag-1 & 419 \\
\hline & 291 & 66,160 & $\pi \rightarrow \pi^{*}$ & $\mathrm{CH}_{2} \mathrm{Cl}_{2}$ & Au-1 & 517 \\
\hline & 251 & 23,260 & $\pi \rightarrow \pi^{*}$ & $\mathrm{H}_{2} \mathrm{O}$ & Ag-1 & $398 / 583^{b}$ \\
\hline & & & & $\mathrm{H}_{2} \mathrm{O}$ & Au-1 & $527^{c}$ \\
\hline \multirow[t]{4}{*}{2} & 458 & 17,320 & $d \rightarrow \pi^{*}$ & $\mathrm{CH}_{2} \mathrm{Cl}_{2}$ & Ag-2 & 423 \\
\hline & 334 & 27,515 & $\pi \rightarrow \pi^{*}$ & $\mathrm{CH}_{2} \mathrm{Cl}_{2}$ & Au-2 & 521 \\
\hline & 290 & 83,640 & $\pi \rightarrow \pi^{*}$ & $\mathrm{H}_{2} \mathrm{O}$ & Ag-2 & $393 / 573^{b}$ \\
\hline & 244 & 26,120 & $\pi \rightarrow \pi^{*}$ & $\mathrm{H}_{2} \mathrm{O}$ & Au-2 & $548^{c}$ \\
\hline \multirow[t]{4}{*}{3} & 463 & 14,670 & $\mathrm{~d} \rightarrow \pi^{*}$ & $\mathrm{CH}_{2} \mathrm{Cl}_{2}$ & Ag-3 & 420 \\
\hline & 342 & 26,530 & $\pi \rightarrow \pi^{*}$ & $\mathrm{CH}_{2} \mathrm{Cl}_{2}$ & Au-3 & 515 \\
\hline & 290 & 65,250 & $\pi \rightarrow \pi^{*}$ & $\mathrm{H}_{2} \mathrm{O}$ & Ag-3 & $390 / 511^{\mathrm{b}}$ \\
\hline & 247 & 23,265 & $\pi \rightarrow \pi^{*}$ & $\mathrm{H}_{2} \mathrm{O}$ & Au-3 & $556^{c}$ \\
\hline \multirow[t]{4}{*}{4} & 453 & 12,300 & $d \rightarrow \pi^{*}$ & $\mathrm{CH}_{2} \mathrm{Cl}_{2}$ & $\mathrm{Ag}-4$ & 416 \\
\hline & 289 & 73,800 & $\pi \rightarrow \pi^{*}$ & $\mathrm{CH}_{2} \mathrm{Cl}_{2}$ & $\mathrm{Au}-4$ & 511 \\
\hline & 250 & 17,170 & $\pi \rightarrow \pi^{*}$ & $\mathrm{H}_{2} \mathrm{O}$ & Ag-4 & 402 \\
\hline & & & & $\mathrm{H}_{2} \mathrm{O}$ & $\mathrm{Au}-4$ & $540-655^{c}$ \\
\hline
\end{tabular}

a In DCM, at $298 \mathrm{~K}$.

b Absorption of both components of the SPB.

c Broad band.

a)

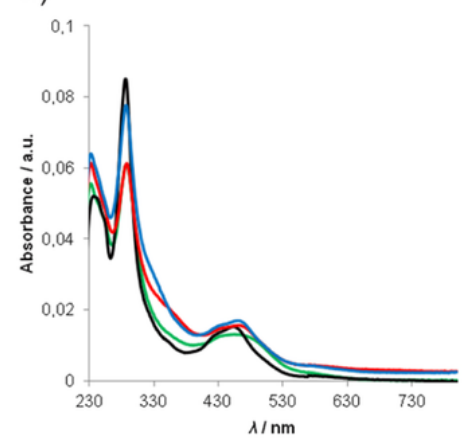

d)

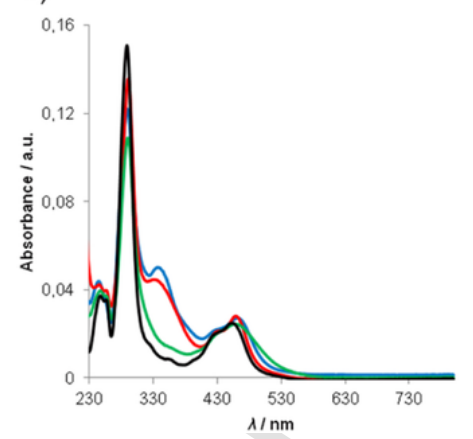

b)

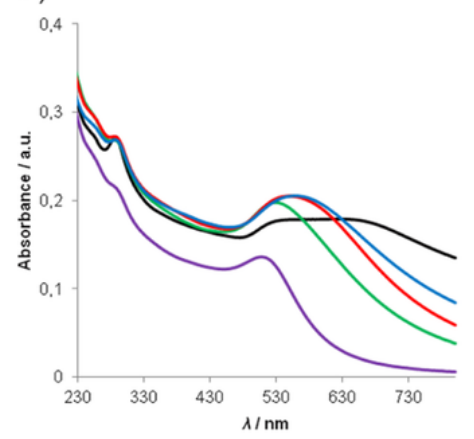

e)

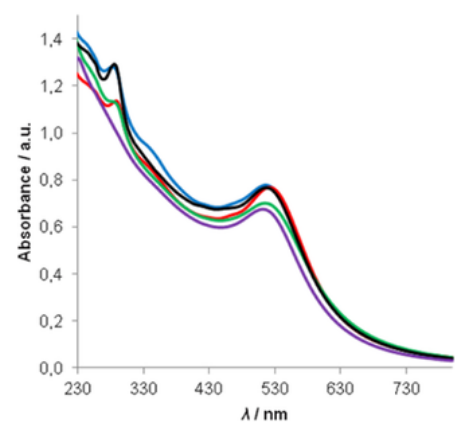

c)

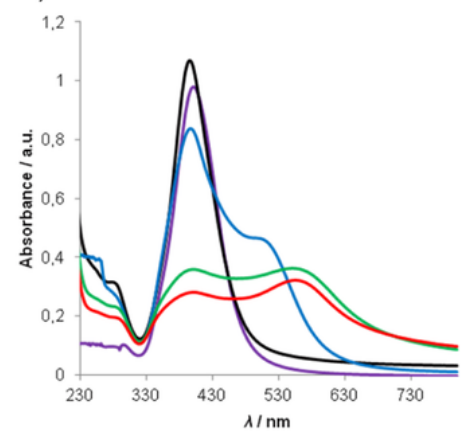

f)

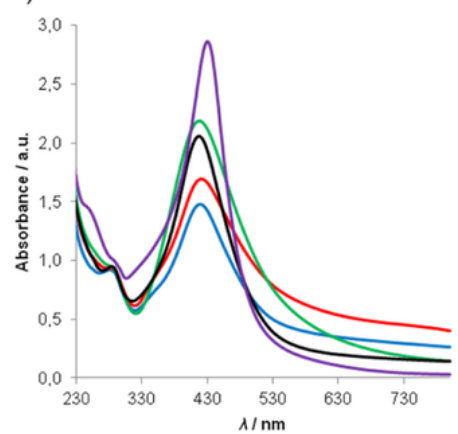

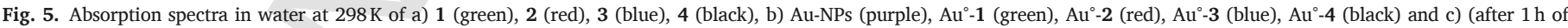

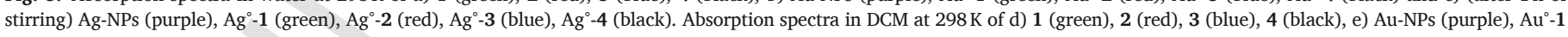

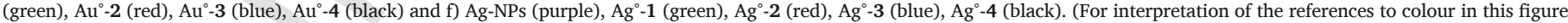
legend, the reader is referred to the web version of this article.)

functionalization induced a small blue shift of the SPB together with a broadening of the peak (Fig. 5f). This broadening of the peak is more important after addition of $\mathbf{2}$, in agreement with the higher polydispersity in size and shape observed by TEM analyses.

Luminescence properties (stationary and time resolved data) of the ruthenium complexes are summarized in Table 2 (Fig. 6 and supporting information). It should be stressed here that a full account of the solvent effect on luminescent properties of Ru-bipyridine complexes is not an easy task because a minimum of three processes, solvent and temperature dependent, are involved: radiative and non-radiative decay from the lowest triplet MLCT emissive state (quenched by the oxygen) as well as the transition to a low lying d-d state $[52,53]$. The energy levels of the MLCT states depend on the polarity of the solvent and influence the transition efficiency to the ground state (radiatively 
Table 2

Luminescence data of free ruthenium complexes in solution (without NPs).

\begin{tabular}{|c|c|c|c|c|c|c|c|c|c|c|c|c|c|c|c|}
\hline & \multicolumn{3}{|c|}{$\lambda_{\mathrm{em}, \max }[\mathrm{nm}]$} & \multicolumn{3}{|c|}{$\Phi_{\mathrm{r}}\left(\mathrm{x} 10^{-2}\right)$} & \multicolumn{3}{|l|}{$\tau[\mathrm{ns}]$} & \multicolumn{3}{|c|}{$k_{\mathrm{r}}\left[10^{4} \mathrm{~s}^{-1}\right]$} & \multicolumn{3}{|c|}{$k_{\mathrm{nr}}\left[10^{4} \mathrm{~s}^{-1}\right]$} \\
\hline & $A C N$ & DCM & Water & $A C N$ & DCM & Water & $A C N$ & DCM & Water & $A C N$ & DCM & Water & $A C N$ & DCM & Water \\
\hline 1 & 648 & 603 & 642 & 1.6 & 9.2 & 3.9 & 860 & 602 & 552 & 1.8 & 15.2 & 7.0 & 114.4 & 150.8 & 174.0 \\
\hline 2 & 625 & 594 & 624 & 2.3 & 12.5 & 9.4 & 771 & 735 & 656 & 2.9 & 17.0 & 14.3 & 126.7 & 119.0 & 138.1 \\
\hline 3 & 617 & 597 & 617 & 0.7 & 11.7 & 7 & 352 & 914 & 570 & 1.9 & 12.8 & 12.2 & 282.1 & 96.6 & 163.1 \\
\hline 4 & 617 & 600 & 623 & 11 & 10.7 & 8.7 & 905 & 575 & 588 & 12.1 & 18.6 & 14.7 & 98.3 & 155.3 & 155.2 \\
\hline
\end{tabular}

a)

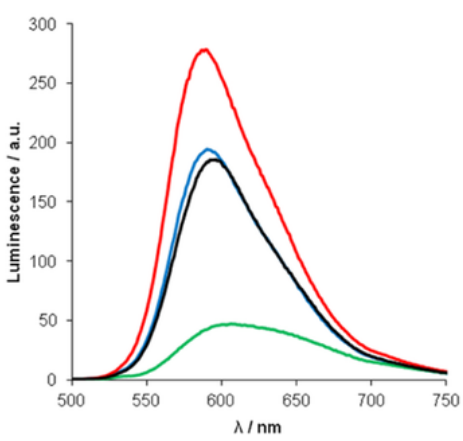

b)

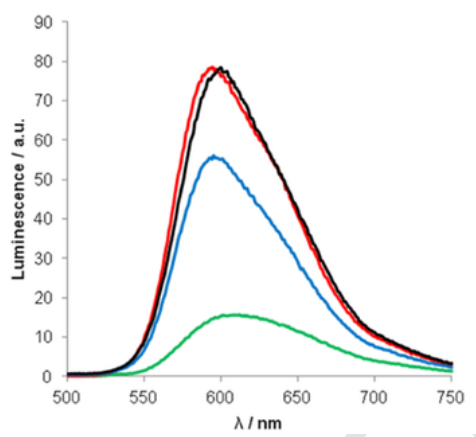

c)

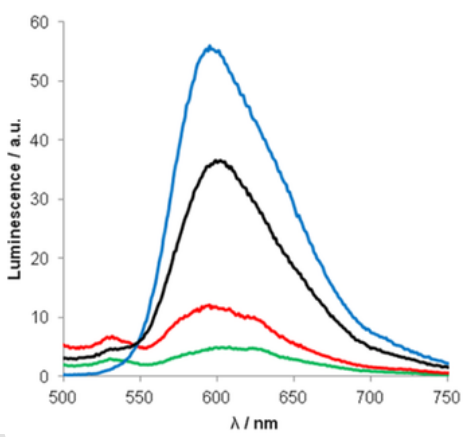

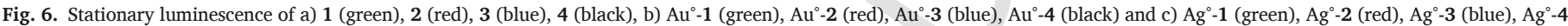
(black) in DCM with $460 \mathrm{~nm}$ excitation. (For interpretation of the references to colour in this figure legend, the reader is referred to the web version of this article.)

and non radiatively) and to the thermally activated d-d states (temperature dependent). It was also shown that $\mathrm{OH}$ vibrations of water play the role of energy acceptors and thus enhance radiationless decay for Ru-bipyridine complexes [54]. Attention should be also paid to the properties of the anchoring group: imidazole groups are known to be electron donor groups and sensitive to $\mathrm{pH}$. Dithioic group is an acid whereas aniline is a weak base and also an electron donor group. Altogether these features will influence the MCLT level and its emissive properties. Therefore luminescence of ruthenium complexes 1-4 was measured in water, acetonitrile and DCM at $298 \mathrm{~K}$ in deoxygenated conditions to try to understand the influence of the polarity and proticity of the solvent. Complex 4 was used as the reference in the whole study to determine luminescence quantum yields of the other complexes $[52,53,55]$. Assuming that the intersystem crossing quantum yield $\left(\Phi_{\mathrm{T}}\right)$ for the formation of the ${ }^{3}$ MLCT triplet state is equal to unity, and knowing the lifetimes $(\tau)$ and emission quantum yield $\left(\phi_{\mathrm{r}}\right)$, the radiative $\left(\mathrm{k}_{\mathrm{r}}\right)$ and non-radiative $\left(\mathrm{k}_{\mathrm{nr}}\right)$ rate constants for the series of complexes can be calculated using the following expressions:

$k_{r}=\frac{\Phi_{r}}{\Phi_{T} \cdot \tau}, k_{n r}=\frac{1}{\tau}-k_{r}$

In DCM a quantum yield about $10 \%$ is found for all the complexes. The lifetime of the MLCT excited state of $\mathbf{1}$ is very close to the value for the reference complex 4 (about $600 \mathrm{~ns}$ ) whereas it is slightly longer for complexes 2 and 3 (735 and 914 ns, respectively). This is mainly due to a lower non radiative constant which can be explained on the basis of a higher energy gap between the lowest excited and the ground state. For complexes 1-3 a general result is a lower luminescence quantum yield combined with a lower radiative constant and a bathochromic shift of the emission maximum of the triplet MCLT excited state from DCM, apolar solvent $(\varepsilon=9.1)$ to acetonitrile (aprotic, polar solvent, $\varepsilon=37.5$ ). In the case of the reference complex 4 , the luminescence quantum yield and radiative constant are almost constant whereas non radiative constant decrease from 155 to $9810^{4} \mathrm{~s}^{-1}$ concomitantly with an increase of its lifetime to $905 \mathrm{~ns}$. This can be ex- plained by a higher energy gap between MLCT and d-d states (bathochromic shift of the emission maximum) and then a lower non radiative constant because of less effective thermal activation of $d-d$ state quenching pathway. In acetonitrile complexes $\mathbf{1}$ and $\mathbf{2}$ present slightly lower lifetime and higher non radiative constant (about $800 \mathrm{~ns}$ and $110.10^{4} \mathrm{~s}^{-1}$ ) in comparison to complex 4 . The main difference is a lower radiative constant which leads to a lower luminescence quantum yield. Longer groups which increase vibrational relaxation pathway and lower energy gap with the ground state is also responsible of these results. Moreover the electron donor effect, which is more important in polar solvent, of different anchoring groups (dithioic groups and imidazole group) changes and reduces the radiative constant as previously observed for methyl- or dimethyl-substitution on bipyridyl ligand [56]. Complex 3 in acetonitrile has the lowest quantum yield which is characterized by a short lifetime ( $350 \mathrm{~ns}$ ), and a non-radiative constant with a maximum value of $282.10^{5} \mathrm{~s}^{-1}$, more than two times the value for the other complexes whereas the radiative constant is quite similar to complexes $\mathbf{1}$ and $\mathbf{2}$ (similar electron donor effect). The former effect comes from the presence of the aniline moiety and the already envisioned photo-induced electron transfer mechanism which is more efficient in polar solvent. Finally the luminescence results in water should be rationalized combining polar solvent influence in addition with specific interactions with the solvent because the anchoring groups are $\mathrm{pH}$ sensitive. Water molecule reduces the electron donor effect of the imidazole group (hydrogen bond with the nitrogen of the imidazole group) and leads for complexes $\mathbf{2}$ and $\mathbf{3}$ to similar radiative constant in comparison to reference complex 4 . For complex 3 the lone pair of the nitrogen can be involved in hydrogen bond and diminish the existence of the photoinduced electron transfer. Therefore similar non radiative constant, luminescence quantum yield and lifetime are found for complexes 2, 3 and 4. For complex 1 a lower radiative constant rate is observed and leads to a lower luminescence quantum yield compare to other complexes in water. The electron donor effect of dithioic acid is quite similar in water and explains this result.

Stationary and time resolved luminescence data after post-functionalization of the metal NPs with a surface coverage of $20 \%$ (see above $\S$ 
Synthesis of the nanocomposites) are summarized in Table 3. Such low coverage percentage was used to avoid intermolecular quenching from different complexes attached to the same NP. Luminescence spectra are similar for the free and bound to NPs complexes, while, as expected from the literature, luminescence of the different complexes are quenched by the NPs (Fig. 6). The reason of such quenching is complex and it was shown that energy transfer and electron transfer can quench the luminescence and that contribution of each process is dependant of the size of NPs; for small size NPs (below $8 \mathrm{~nm}$ ) the electron transfer quenching is dominant $[43,46,57]$. It was also mentioned that the quenching for chromophores bound to NPs can be due to a drastic decrease of the radiative constant rate [58]. Analysis of Fig. 7 and Table 3 leads to several comments: 1) bound complexes (1, 2 and 3) show a higher luminescence quenching compare to complex 4 which interacts with NPs only through electrostatic interactions, 2) the luminescence quenching is higher in polar solvent (almost 99\% in water) which is characteristic of a quenching by electron transfer, 3) the quenching is more important for complex $\mathbf{3}$ than complex $\mathbf{2}$ and this result can be explained by a longer distance between the NPs and the ruthenium centres, 4) pyridine and aniline anchoring groups show a higher quenching which is due to an additional specific photo-induced electron transfer which increases in the vicinity of the NPs, 5) the quenching is more important for Ag-NPs due to a lower oxidation potential of Ag-NPs compared to Au-NPs and this reinforces a mechanism by electron transfer. The luminescence lifetime in DCM (the quenching was too strong to be able to measure luminescence lifetime in water) is multiexponential in agreement with the existence of several quenching processes and was calculated by the mean lifetime procedure [59]. The mean lifetime is about $10 \mathrm{~ns}$ (about $500 \mathrm{~ns}$ for free complexes), in agreement with the strong decrease of luminescent quantum yield. As the luminescence is less quenched for Au-NPs compared to Ag-NPs, the mean lifetime is longer for Au-NPs. An interesting result is that while the luminescence quantum yield of the post-functionalized NPs is the lowest for complexes $\mathbf{2}$ and $\mathbf{3}$, the mean lifetime is slightly longer compared to complexes 1 and 4, a slower back electron transfer (longer distance between the NPs and the ruthenium centres) can be involved for the long component. It should be emphasized here that no long component was found for free complexes (few hundreds of nanosec-

Table 3

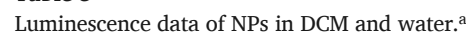

\begin{tabular}{|c|c|c|c|c|c|c|}
\hline Compounds & $\begin{array}{l}\tau[\mathrm{ns}] \text { with Au- } \\
\text { NPs in DCM }\end{array}$ & $\begin{array}{l}\tau \text { [ns] with Ag- } \\
\text { NPs in DCM }\end{array}$ & $\begin{array}{l}\text { Luminescence Quenching \% } \\
\text { of Au-NPs in DCM }\end{array}$ & $\begin{array}{l}\text { Luminescence Quenching \% } \\
\text { of Ag-NPs in DCM }\end{array}$ & $\begin{array}{l}\text { Luminescence Quenching \% } \\
\text { of Au-NPs in water }\end{array}$ & $\begin{array}{l}\text { Luminescence Quenching \% } \\
\text { of Ag-NPs in water }\end{array}$ \\
\hline 1 & 9.97 & 6.98 & 68.8 & 86.8 & 99.3 & 91.7 \\
\hline 2 & 10.51 & 8.07 & 75.4 & 94.3 & 99.8 & 98.1 \\
\hline 3 & 12.19 & 10.36 & 78.1 & 97.6 & 99.8 & 97.8 \\
\hline 4 & 6.57 & 5.73 & 59 & 80.8 & 87 & 85.9 \\
\hline
\end{tabular}

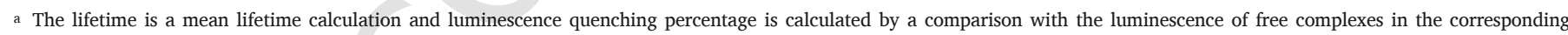
solvent.

a)

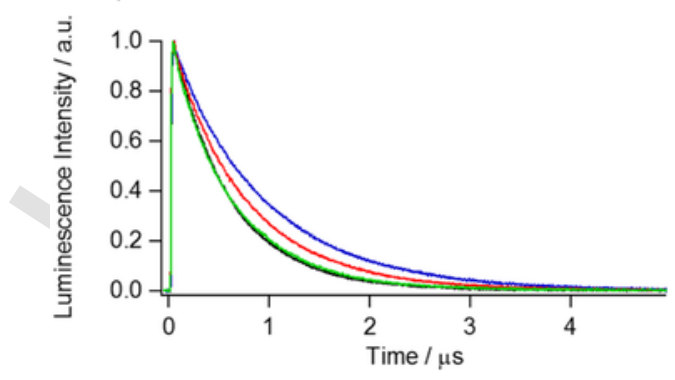

onds) and therefore it confirms that the complexes are either attached (complexes 1, 2 and 3) or in the vicinity of the NPs (complex 4). Our experiments lead to the main conclusion that electron transfer should be the main parameter in the strong luminescent quenching of complexes attached directly to the NPs. This agrees with previous reports about NPs with sizes below $8 \mathrm{~nm}$. An interesting result is that a longer distance between the NPs and the ruthenium complexes leads to a longer mean lifetime.

$\bar{\tau}=\frac{\int_{0}^{\infty} t * I(t) * d t}{\int_{0}^{\infty} I(t) * d t}$

\subsection{Electrochemistry}

It must be emphasized that the as-prepared post-functionalized Au-NPs and Ag-NPs do not exhibit any electrochemical response when dispersed in DCM solution, due to their too low concentration in electroactive complex (ca. $1 \mu \mathrm{M}$ ). In order to record cyclic voltammetry on these nanocomposites, a modified synthetic procedure had to be implemented with the formation of NPs directly in a $2.10^{-4} \mathrm{M}$ solution of the complex [34]. Fig. 8 displays the electrochemical behaviour, on gold electrode, of 1 either free or grafted onto Au-NPs or Ag-NPs. The oxidation peak at $+1 \mathrm{~V}$ can be attributed to the $\mathrm{Ru}^{\mathrm{II}} / \mathrm{Ru}^{\mathrm{III}}$ redox couple. From the shape of the CV it can be noticed that the oxidized form of the free complex tends to strongly adsorb on the electrode surface: the diffusion tail visible on the forward peak totally vanishes on the backward peak that displays a symmetrical shape. The same redox couple grafted on Au-NPs or Ag-NPs displays a very different behaviour: while oxidation takes place at nearly the same potential as for the free complex, the corresponding reduction $\left(\mathrm{Ru}^{\mathrm{III}} \rightarrow \mathrm{Ru}^{\mathrm{II}}\right)$ is no more visible. The same behaviour is observed for 2 , and whatever the metallic core of the NP. It is likely that a passivation phenomenon occurs at the electrode surface upon oxidation of the complex. This was confirmed by rotating disk electrode (RDE) experiments that are more likely to maintain the nanocomposites in solution to the detrimental of adsorption 


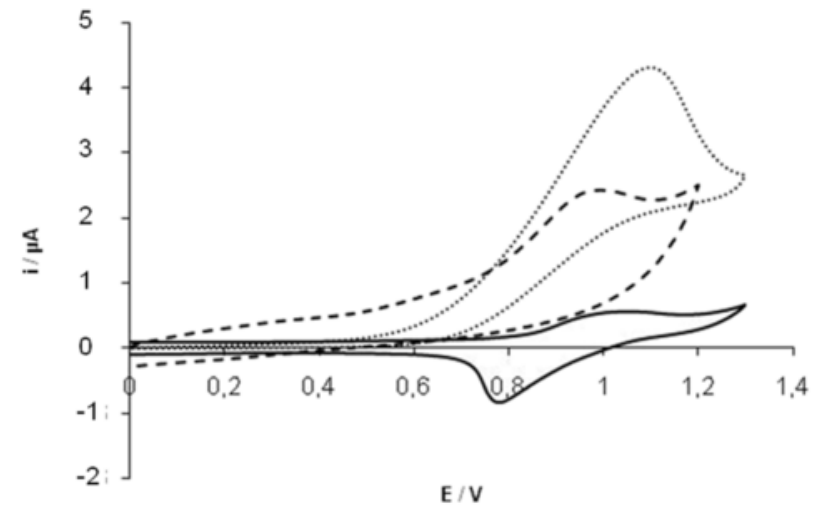

Fig. 8. CVs in DCM of free complex 1 (solid line), complex 1 grafted on Au-NPs (dotted line) and complex 1 grafted on Ag-NP (dashed line).

processes (Fig. 9a): the limiting current does not follow the expected linear dependence with the square root of the rotation rate. The reason for this passivation phenomenon is not fully elucidated but it might be related to the high concentration of NPs required to maintain the concentration in ruthenium complexes to sufficiently high values to be detected, and to an aggregation process induced by the charge increase on the complex (Scheme 4). When the nanoparticles are adsorbed on the electrode surface, the electroactive species become less accessible, leading to successive scans showing no more signal until the electrode surface is cleaned by polishing.

The forward peak current $\left(\mathrm{I}_{\mathrm{p}}\right)$ variation with the scan rate shows a square root dependence characteristic of diffusion limited process both for free complex or nanoparticle functionalized ones but current values do not scale with the diffusion coefficient estimated from Stokes-Einstein equation. As already observed an exalted current due to charge storage by the nanoparticles is recorded [34]. Indeed, based on Randles-Sevcik equation [60], one obtains for the ratio of the apparent diffusion coefficients:

$$
\frac{D_{N P}}{D_{\text {free }}}=\left(\frac{\alpha_{N P} C_{\text {free }}}{\alpha_{\text {free }} C_{N P}}\right)^{2}
$$

where $\alpha$ is the slope of the $\mathrm{I}_{\mathrm{p}} \mathrm{vs} . \mathrm{v}^{1 / 2}$ straight line, $C$ is the bulk concentration in electroactive species, and 'NP' and 'free' indexes correspond to the complex on nanoparticle and in solution, respectively. From experimental data recorded with the same concentration in free and bound ruthenium complexes, one obtains a ratio of nearly 400, which is by far larger than what can be predicted from the relative sizes of NP and free complex. The reason for this extra-current is double layer charging of the nanoparticle [61], but modification of the electroactive area involving physisorption of nanoparticles is likely to be also involved. To investigate further this phenomenon, we performed chronoamperometry at various final potentials corresponding to the foot of the current-potential wave. In that potential range, mainly metallic nanoparticle cores are concerned by the electrochemical reaction. The results (Fig. 9b) show a Cottrellian behaviour, as expected for a diffusion limited current associated with species in solution, with a slope that depends on the electrode potential (Fig. 9c). Assuming that no modification of the electroactive area of the electrode occurs in that potential range (i.e. adsorption occurs only when the complex is fully oxidized), one can relate this slope to both the (starting) faradaic oxidation of the ruthenium centres and the double layer charging of the nanoparticle [62], according to the following equation derived from Murray's analysis [63]:

$i=A \sqrt{\frac{D_{N P}}{\pi}} C_{N P}\left(F \theta_{R u} \frac{1}{1+\exp \left(-f\left(E-E^{\circ}\right)\right)}+A_{N P} N_{A} C_{N P}^{d l}\left(E-E_{i}\right)\right) \frac{1}{\sqrt{t}}$

where $A$ is the electrode area, $A_{\mathrm{NP}}$ is the nanoparticle area, $N_{\mathrm{A}}$ is the Avogadro number, $\theta_{\mathrm{Ru}}$ is the ruthenium per nanoparticle ratio, $F$ is the Faraday constant $(f=F / R T), E_{\mathrm{i}}$ and $E$ are the initial and final potentials, $E^{\circ}$ is the standard potential for $\mathrm{Ru}{ }^{\mathrm{II}} / \mathrm{Ru}^{\mathrm{III}}$ and $C_{N P}{ }^{\mathrm{dl}}$ is the double layer capacitance per unit area for the nanoparticle. In Eq. (1), the first

a)

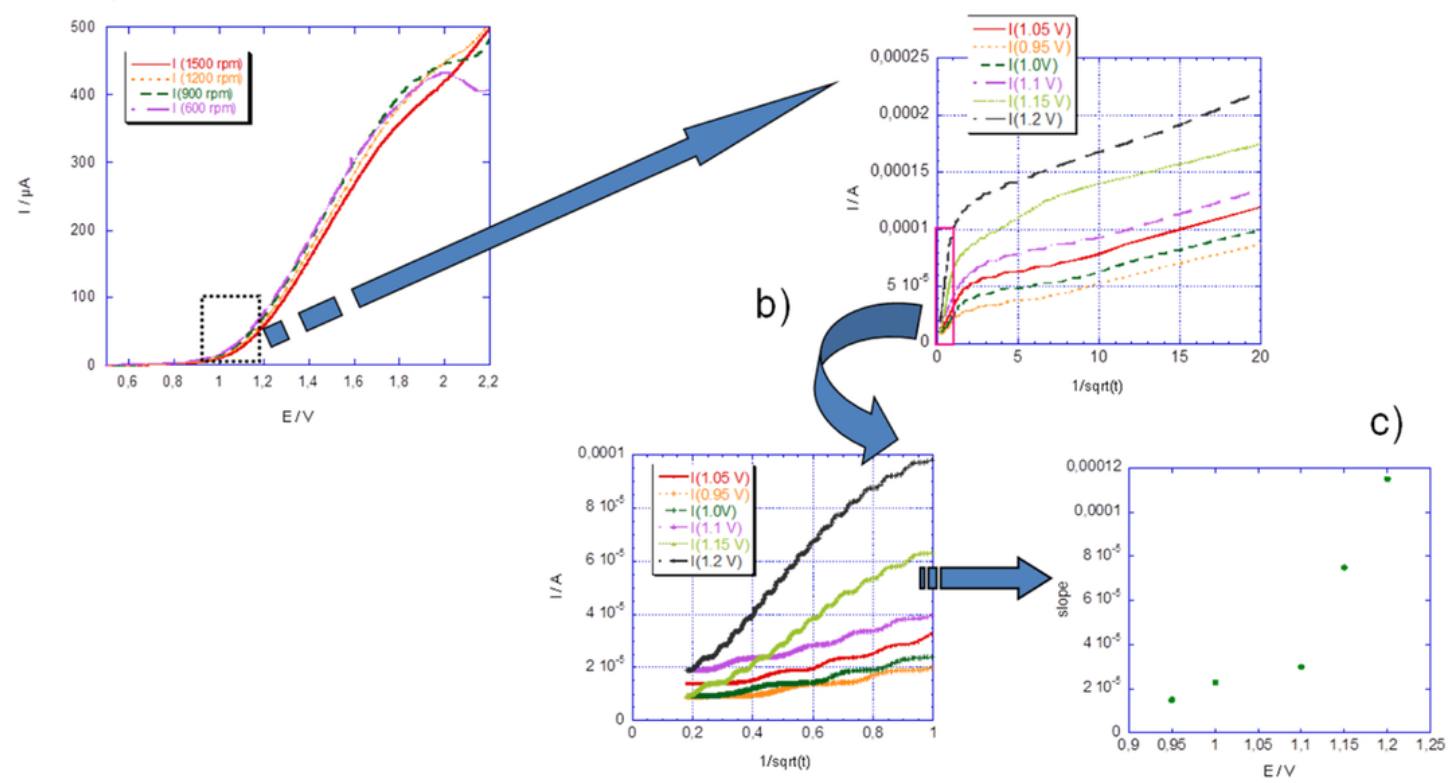

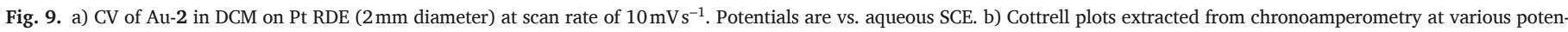

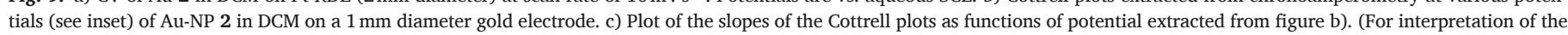
references to colour in this figure legend, the reader is referred to the web version of this article.) 
term of the sum is the faradaic oxidation of ruthenium which can be neglected (at least for the lowest values of the investigated potentials in the chronoamperometric measurements) taking into account the standard potential drawn from Fig. 9a. In that model, the curve of Fig. 9c should display the variation of the double layer charging of the nanoparticle with the applied potential (second term in Eq. (1)). From the values of the various parameters involved in Eq. (1) $\left(D_{N P}=10^{-6} \mathrm{~cm}^{2} \mathrm{~s}^{-1}\right.$, $A_{N P}=210^{-13} \mathrm{~cm}^{2}$ ) and the measured slope, one can draw an estimation of the value of the double layer capacitance $C_{N P}{ }^{d l}$ equal to $10 \mu \mathrm{F} \mathrm{cm}^{-2}$, in agreement with reported values for gold functionalized clusters [63].

Finally Table 4 displays the redox potentials deduced from CV for the various free and bound complexes. The oxidation potentials are all in the same range for Au-NPs and Ag-NPs and close to the value of the free complex excepted for 3 (see Table 4). In this latter case, the difference can arise from the aniline group, which can be oxidized in the free complex at lower potential than the ruthenium centre but not in the nanocomposite where it is involved in the NP surface functionalization. Further investigations are in progress on the controlled deposition of these functionalized nanoparticles on metallic surfaces to highlight some contributions from the linker or the nanoparticle size on the electrochemical features of the attached redox moiety.

\section{Conclusion}

A series of three ruthenium (II) complexes with different pendant groups, namely pyridine, aniline and carboxydithioic acid, have been reacted with preformed Au-NPs and Ag-NPs in water and in dichloromethane. Their reactivity and properties have been compared to the ones of the model $\left[\mathrm{Ru}(\mathrm{bpy})_{3}\right]^{2+}$ complex which does not own any anchoring group. We showed that water was not an appropriate solvent to obtain stable colloidal solutions. We also showed that the three polypyridyl ruthenium complexes possessing anchoring groups can drastically modify the size as well as the morphology of preformed Ag-NPs. Comparatively, alterations of preformed Au-NPs are less important using the same complexes, due to higher oxidation potentials. The strength of the interaction between the anchoring group and the surface of NPs influenced the size, shape and stability of the final nanocomposites. Polar solvent like water induced aggregation and lead to unstable nanocomposites. Stationary and time resolved luminescence of grafted nanocomposites (1, 2 and 3 ) showed that the luminescence of complexes were completely quenched (lifetime and emission quantum yield) in water by electron transfer processes. Current enhancement due to charge storage by the metal NPs and passivation phenomena of the electrode surface have also been characterized. The current enhancement can be associated with double layer charging of the nanoparticle cores. An interesting result is that while the NPs luminescence quantum yield is the lowest for pyridine and aniline anchor-

Table 4

Oxidation potentials deduced from $\mathrm{CV}$ experiments. Potentials refer to $\mathrm{Ag}^{+} / \mathrm{Ag}$ reference.

\begin{tabular}{ll}
\hline Compound & $E_{\mathrm{p}, \mathrm{ox}} / \mathrm{V}\left(\text { scan rate } / \mathrm{mV} \mathrm{s}^{-1}\right)^{\mathrm{a}}$ \\
\hline $\mathbf{1}$ & $1.05(100)$ \\
$\mathbf{2}$ & $0.97(20)$ \\
$\mathbf{3}$ & $0.76(20)$ \\
$\mathbf{4}$ & $0.96[64]$ \\
$\mathbf{A u}^{\circ}-\mathbf{1}$ & $1.10(100)$ \\
$\mathbf{A g}^{\circ}-\mathbf{1}$ & $0.99(100)$ \\
$\mathbf{A u}^{\circ}-\mathbf{2}$ & $0.98(20)$ \\
$\mathbf{A g}^{\circ}-\mathbf{2}$ & $0.94(20)$ \\
$\mathbf{A g}^{\circ}-\mathbf{3}$ & $0.93(20)$ \\
$\mathbf{A u}-\mathbf{4}$ & $0.90(20)$ \\
$\mathbf{A g}^{\circ}-4$ & $0.92(20)$ \\
\hline
\end{tabular}

a All potentials are measured in acetonitrile $+\mathrm{TBAPF}_{6} 0.1 \mathrm{M}$ dried over molecular sieves. ing groups, the mean lifetime is slightly longer. A slower back electron transfer (longer distance between the NPs and the ruthenium centres) can be involved for the long component. Moreover, it should be emphasized here that no long component from free complexes were found (few hundreds of nanoseconds) and therefore it confirms that the complexes are attached (complexes 1, 2 and 3 ) or in the vicinity of the NPs (complex 4). Interpretation of the optical properties' measurements may be also done from a broader perspective. In particular, the widths and the spectral positions of the observed plasmon resonances in absorbance may be connected with the shape of the nanoparticles, the size of their aggregates and the permittivity of the solvents and ligands. Similarly, fluorescence quenching and enhancement may have electrodynamics nature rather than being solely connected with charge transfer processes. To get a better knowledge of the nature of the quenching processes and to be able to quantify them, ultrafast spectroscopy studies are needed (femtosecond and picosecond time resolved experiments) and are under progress.

\section{Experimental section}

\subsection{Chemicals}

All reagents and solvents were purchased from Aldrich, Alfa Aesar or SDS and used as received without further purification. All aqueous solutions were prepared using ultrapure water purified with a Millipore- $\mathrm{Q}^{+}$system. 1,10-phenanthroline-5,6-dione (phendione) [65], $\left[\left(\right.\right.$ bpy ${ }_{2} \mathrm{Ru}$ (phendione) $] \mathrm{Cl}_{2} \quad$ (7) $[66], \quad\left[(\mathrm{bpy})_{2} \mathrm{RuCl}_{2}\right] \quad$ (8) [67], $\left[\mathrm{Ru}(\mathrm{bpy})_{3}\right](\mathrm{X})_{2} \quad\left(\mathrm{X}=\mathrm{Cl}^{-} \quad\left(4 . \mathrm{Cl}_{2}\right)\right.$ or $\left.\mathrm{PF}_{6}{ }^{-} \quad\left(4 .\left(\mathrm{PF}_{6}\right)_{2}\right)\right) \quad$ [53], [(bpy) ${ }_{2} \mathrm{Ru}\left(4,4^{\prime}\right.$-bis-(chloromethyl)-2,2'-bipyridine) $\mathrm{Cl}_{2}\left(5 . \mathrm{Cl}_{2}\right)$ [39], and [(bpy) ${ }_{2} \mathrm{Ru}((4$-aminophenyl)imidazo[4,5-f] $[1,10]$ phenanthroline $)]\left(\mathrm{PF}_{6}\right)_{2}$ $\left(3 .\left(\mathrm{PF}_{6}\right)_{2}\right)$ [68] were synthesized following procedures previously reported in the literature, without modification and with similar yields.

\subsection{Instrumentation and methods}

ESI-MS measurements were carried out with an API 3000 (ESI/MS/ MS) PE-SCIEX triple quadrupole mass spectrometer and a HP 5989B single quadrupole mass spectrometer equipped with an electrospray source from Analytica of Branford. Both instruments were operated in the positive ion mode. For the API 3000 (ESI/MS/MS) PE SCIEX triple quadrupole mass spectrometer, the experiments were performed either by direct infusion with a syringe pump with a flow rate of $10 \mu \mathrm{l} \cdot \mathrm{min}^{-1}$ or by flow injection acquisition with a flow rate of $200 \mu \mathrm{l} \cdot \mathrm{min}^{-1}$. Standard experimental conditions were the followings: sample concentration: $10^{-4}$ to $10^{-5} \mathrm{M}$, nebulising gas $\mathrm{N}_{2}: 7$ units flow rate on a range of 10, ion spray voltage: $-5.00 \mathrm{kV}$, temperature: $200-400^{\circ} \mathrm{C}$, declustering potential: $-20 \mathrm{~V}$, focusing potential: $-200 \mathrm{~V}$, entrance potential: $10 \mathrm{~V}$. UV/Vis absorption spectra and luminescence spectra were recorded on a Varian CARY 5000 and Fluoromax 3 spectrophotometer. Luminescence decay curves, in the nanosecond domain, were obtained with a time-correlated single- photon-counting method using a titanium-sapphire laser pumped by an argon ion laser (Tsunami, by Spectra-Physics, $82 \mathrm{MHz}, 1$ ps pulse width, repetition rate lowered to $4 \mathrm{MHz}$ thanks to a pulse-peaker, a doubling crystals was used to reach $495 \mathrm{~nm}$ excitation) [69]. Luminescence decays in the microsecond range were recorded using a laser flash photolysis apparatus [70]. Excitation pulses $(460 \mathrm{~nm}$, fwhm $4 \mathrm{~ns}, 1 \mathrm{~mJ}, 0.5 \mathrm{~Hz}$ ) were provided by a $10-\mathrm{Hz} \mathrm{Nd}$ :YAG laser (Continuum Surelite II) coupled to an OPO (Continuum Panther EX OPO) and SH05 shutter (Thorlabs). The emitted light was collected at $90^{\circ}$, dispersed by a monochromator (Horiba Jobin-Yvon, iHR320) and analyzed with a photomultiplier (R1477-06, Hamamatsu) coupled to a digital oscilloscope (LeCroy 454, 500 MHz). Synchronization of excitation pulses and acquisition time was secured with PCI-6602 8 Channel counter/timer (National Instruments). The experiment was controlled 
by the home made software written in LabView environment. The recorded traces were averaged for several pulses and repeated for different wavelengths. The deconvolutions of the individual decays with experimentally measured instrument response function (IRF) lead to $4 \mathrm{~ns}$ time resolution. Single wavelengths as well as global analyses of the transient absorption data were performed using Igor Pro $6.20 .{ }^{1} \mathrm{H}$ NMR spectra were recorded at room temperature in $5 \mathrm{~mm}$ o.d. tubes on a Bruker Avance 300 spectrometer equipped with a QNP probe head. Elemental analyses were performed by the "Service Central d'Analyse du CNRS", Vernaison (France). Electrochemical measurements were performed in a three electrode cell equipped with a $1 \mathrm{~mm}$ diameter platinum disk as the working electrode, platinum wire as the counter electrode and $\mathrm{Ag}^{+}(0.01 \mathrm{M}) / \mathrm{Ag}$ as the reference electrode (SCE for RDE experiments). The reference potential of $\mathrm{Ag}^{+} / \mathrm{Ag}$ was checked vs. ferrocene as recommended by IUPAC $\left(\mathrm{E}^{\circ}(\mathrm{Fc})=+86 \mathrm{mV}\right)$ [71]. The supporting electrolyte was tetrabutylammonium hexafluorophosphate (Fluka, puriss) and the solutions were deaerated by argon bubbling prior to each experiment. Cyclic voltammograms were recorded with a 600 $\mathrm{CH}$ Instruments potentiostat connected to a PC. The size of the NPs was obtained using transmission electron microscope (TEM) images treatment and dynamic light scattering (DLS). TEM images were obtained with a JEOL JEM 100 CXII transmission electron microscope at an accelerating voltage of $100 \mathrm{kV}$, a Zeiss EM902 at $70 \mathrm{kV}$ and for high resolution measurements with a JEOL JEM 2010 at $200 \mathrm{kV}$. DLS measurements were performed with a MALVERN Zetasizer nanoZS.

\section{3. $\left[(\text { bpy })_{2} R u\left(4,4^{\prime}\right.\right.$-(carboxydithioic acid)-2,2'-bipyridine $\left.)\right]\left(P F_{6}\right)_{2}(1$. $\left.\left(P F_{6}\right)_{2}\right)$}

Elemental sulphur $(69 \mathrm{mg}, 2.15 \mathrm{mmol}$ ) and of sodium methanolate (116 mg, $2.15 \mathrm{mmol})$ were placed in anhydrous methanol $(50 \mathrm{~mL})$ under argon atmosphere and refluxed until elemental sulphur was completely dissolved (ca. $2 \mathrm{~h}) .5 .\left(\mathrm{PF}_{6}\right)_{2}(512 \mathrm{mg}, 0.54 \mathrm{mmol})$ was then added and the reaction mixture was refluxed overnight. The solvent was then evaporated and the red solid obtained was dissolved in water. Acidification with diluted $\mathrm{HCl}$ led to the precipitation of the complex, which was filtered and washed several times with water. The product was dissolved in a minimum amount of acetonitrile. Addition of a saturated aqueous solution of $\mathrm{NH}_{4} \mathrm{PF}_{6}$ led to the precipitation of $1 .\left(\mathrm{PF}_{6}\right)_{2}(465 \mathrm{mg}, 86 \%)$ as a dark orange solid, which was collected by filtration, washed with $\mathrm{H}_{2} \mathrm{O}$ and dried under vacuum. ${ }^{1} \mathrm{H}$ NMR $\left(300 \mathrm{MHz}, \mathrm{CD}_{3} \mathrm{CN}\right): \delta=8.49(\mathrm{~m}, 6 \mathrm{H})$, $8.05(\mathrm{~m}, 6 \mathrm{H}), 7.72(\mathrm{~m}, 4 \mathrm{H}), 7.39 \mathrm{ppm}(\mathrm{m}, 6 \mathrm{H})$; elemental analysis calcd (\%) for $\mathrm{C}_{36} \mathrm{H}_{35} \mathrm{~F}_{12} \mathrm{~N}_{7} \mathrm{O}_{2} \mathrm{P}_{2} \mathrm{RuS}_{4}\left(1 .\left(\mathrm{PF}_{6}\right)_{2} \cdot\left(\mathrm{CH}_{3} \mathrm{OH}\right)_{2} \cdot\left(\mathrm{CH}_{3} \mathrm{CN}\right)\right)$ : C 38.7, $\mathrm{H}$ 3.2, N 8.7, S 11.5; found: C 38.5, H 2.8, N 8.4, S 11.3.

\section{4. $\left[(\text { bpy })_{2} R u((4-(4-p y r i d i n y l) p h e n y l) i m i d a z o[4,5-\right.$ $f][1,10]$ phenanthroline $)]\left(P F_{6}\right)_{2}\left(2 .\left(P F_{6}\right)_{2}\right)$}

$7 \quad(0.115 \mathrm{~g}, \quad 0.16 \mathrm{mmol}), \quad 4-(4-p y r i d i n y l) b e n z a l d e h y d e \quad(0.040 \mathrm{~g}$, $0.2 \mathrm{mmol})$ and $\mathrm{NH}_{4} \mathrm{OAc}(0.3 \mathrm{~g}, 4 \mathrm{mmol})$ were stirred at reflux overnight in glacial acetic acid $(30 \mathrm{~mL})$. After cooling, the dark red solution was concentrated under reduced pressure to ca. $5 \mathrm{~mL}$ and the crude product was precipitated using a concentrated aqueous solution of ammonium hexafluorophosphate. The product was purified by dissolution in acetonitrile and precipitation by slow addition of diethyl ether. 2. $\left(\mathrm{PF}_{6}\right)_{2}$ was filtered, washed with ethanol $(2 \times 10 \mathrm{~mL})$, diethyl ether $(2 \times 10 \mathrm{~mL})$ and dried under vacuum $(0.078 \mathrm{~g}, 78 \%) .{ }^{1} \mathrm{H}$ NMR (DMSO- $\left.d_{6}\right): \delta=13.5(\mathrm{~s}, 1 \mathrm{H}), 8.76(\mathrm{~d}, 2 \mathrm{H}), 8.54(\mathrm{~m}, 6 \mathrm{H}), 8.27(\mathrm{~d}, 2 \mathrm{H})$, $8.15(\mathrm{~m}, 2 \mathrm{H}), 8.12(\mathrm{~m}, 2 \mathrm{H}), 8.05(\mathrm{~m}, 4 \mathrm{H}), 7.93(\mathrm{~m}, 2 \mathrm{H}), 7.85(\mathrm{~m}, 2 \mathrm{H})$, 7.78 (m, 2H), 7.61 (m, 2H), 7.46 (dd, 2H), 7.22 ppm (dd, 2H); ESI-MS: $\mathrm{m} / z$ (positive mode): $393.7\left([M]^{2+} / 2\right.$ ) elemental analysis calcd (\%) for
$\mathrm{C}_{44} \mathrm{H}_{31} \mathrm{~N}_{9} \mathrm{RuP}_{2} \mathrm{~F}_{12}\left(2 .\left(\mathrm{PF}_{6}\right)_{2}\right)$ : C 49.03, H 3.09, N 11.71, Ru 9.39, P 5.75, F 21.17; found: C 49.26, H 2.97, N 11.76, Ru 9.23, P 5.83, F 21.32.

\subsection{Synthesis of $1 . \mathrm{Cl}_{2}, 2 . \mathrm{Cl}_{2}$ and $3 . \mathrm{Cl}_{2}$}

Hexafluorophosphate salts of $\mathbf{1}, \mathbf{2}$ and $\mathbf{3}$ were converted to the corresponding chloride salts by dissolution of the hexafluorophosphate salts in acetone and addition of an excess of tetrabutylammonium chloride [72].

Preparation of the starting hydrosols of Au-NPs and Ag-NPs:

$A u$-NPs in water: Aqueous solutions of $\mathrm{HAuCl}_{4} \cdot 3 \mathrm{H}_{2} \mathrm{O}(1 \mathrm{~mL}, 0.025 \mathrm{M})$ and sodium citrate $(1 \mathrm{~mL}, 0.025 \mathrm{M})$ were poured into $98 \mathrm{~mL}$ of water. Then, a cooled aqueous solution of $\mathrm{NaBH}_{4}(1.3 \mathrm{~mL}, 0.1 \mathrm{M})$ was added dropwise. During the addition, the colour of the mixture turned to red-wine. The mean diameter of Au-NPs determined from TEM images was $2.5 \mathrm{~nm}$. $\mathrm{Ag}$-NPs in water: Aqueous solutions of $\mathrm{AgNO}_{3}(2.36 \mathrm{~mL}, 0.01 \mathrm{M})$ and sodium citrate $(1 \mathrm{~mL}, 0.025 \mathrm{M})$ were poured into $250 \mathrm{~mL}$ of water. Then, a cooled aqueous solution of $\mathrm{NaBH}_{4}(4.2 \mathrm{~mL}, 0.1 \mathrm{M})$ was added dropwise, followed by another addition of an aqueous solution of $\mathrm{NaBH}_{4}$ $(0.3 \mathrm{~mL}, 0.1 \mathrm{M})$ ten minutes later. During the addition, the colour of the mixture turned yellow. The mean diameter of Ag-NPs, calculated from TEM images, was $2.5 \mathrm{~nm}$.

\subsection{Post-functionalization of $\mathrm{Au}-\mathrm{NPs}$ and $\mathrm{Ag}$-NPs hydrosols using the} chloride salts of ruthenium complexes 1, 2, 3 and 4

As already mentioned in the article, the amount of ruthenium complexes added to post-functionalize the metal NPs was about $20 \%$ of the theoretical amount needed for the maximal covering. This low concentration of ruthenium complexes was chosen to limit the communication between them once attached to the surface of the NPs. The surface of the Au-NPs and Ag-NPs was $19.63 \mathrm{~nm}^{2}$, considering the mean diameter of $2.5 \mathrm{~nm}$ determined using TEM images. The footprint area of the ruthenium complexes was determined to be $\sim 1 \mathrm{~nm}^{2}$. As a result, a very simple calculation led to a maximum number of ruthenium complexes per particle of 19. The post-functionalization was realized using either $10 \mathrm{~mL}$ of the previous hydrosol of Au-NPs or $26.5 \mathrm{~mL}$ of the previous hydrosol of Ag-NPs that were further diluted with water to obtain a final volume of $60 \mathrm{~mL}$. Both solutions contained the same amount of metal NPs (5.26 $10^{-9} \mathrm{~mol}$ using a tight-packed spherical model [73]). Then aqueous solutions of $1 . \mathrm{Cl}_{2}\left(70 \mu \mathrm{L}, 2.6510^{-4} \mathrm{M}\right), \mathbf{2} . \mathrm{Cl}_{2}\left(70 \mu \mathrm{L}, 2.3210^{-4} \mathrm{M}\right), \mathbf{3}^{-\mathrm{Cl}_{2}}$ $\left(70 \mu \mathrm{L}, 2.5110^{-4} \mathrm{M}\right)$ or $4 . \mathrm{Cl}_{2}\left(70 \mu \mathrm{L}, 2.910^{-4} \mathrm{M}\right)$ were added to each hydrosol and the resulting solutions were stirred for $48 \mathrm{~h}$ at $25^{\circ} \mathrm{C}$ before analysis.

Preparation of the starting DCM solutions Au- and Ag-NPs:

Au-NPs in DCM: $\mathrm{HAuCl}_{4} .3 \mathrm{H}_{2} \mathrm{O}\left(0.010 \mathrm{~g}, 2.510^{-5} \mathrm{~mol}\right)$ and 1-hexadecylamine $\left(0.200 \mathrm{~g}, 8.310^{-4} \mathrm{~mol}\right)$ were dissolved in DCM $(10 \mathrm{~mL})$. The final volume of the solution was adjusted to $60 \mathrm{~mL}$ using DCM. A cooled aqueous solution of $\mathrm{NaBH}_{4}(400 \mu \mathrm{L}, 1 \mathrm{M})$ was then added and the resulting mixture was stirred for $1 \mathrm{~h}$. The water phase was removed using a micropipette. The mean diameter of Au-NPs determined from TEM images was $\sim 3 \mathrm{~nm}$.

Ag-NPs in DCM: $\mathrm{AgNO}_{3}\left(0.004 \mathrm{~g}, 2.510^{-5} \mathrm{~mol}\right)$ and 1-hexadecylamine $\left(0.075 \mathrm{~g}, 3.110^{-4} \mathrm{~mol}\right)$ were dissolved in DCM $(5 \mathrm{~mL})$. Oleic acid $(10 \mu \mathrm{L})$ was then added and the volume of the solution was adjusted to $60 \mathrm{~mL}$ using DCM. A cooled aqueous solution of $\mathrm{NaBH}_{4}(100 \mu \mathrm{L}, 1 \mathrm{M})$ was then added and the resulting mixture was stirred for $1 \mathrm{~h}$. The water phase was removed using a micropipette. The mean diameter of Ag-NPs determined from TEM images was determined to be $\sim 3 \mathrm{~nm}$. 


\subsection{Post-functionalization of Au-NPs and Ag-NPs in DCM using the hexafluorophosphate salts of ruthenium complexes 1, 2, 3 and 4}

The same approach was used in DCM and in water with the aim of adding an amount of ruthenium complexes of about $20 \%$ of the theoretical amount needed for the maximum covering. DCM solutions of 1. $\left(\mathbf{P F}_{6}\right)_{2}\left(70 \mu \mathrm{L}, 2.3210^{-4} \mathrm{M}\right)$, 2. $\left(\mathbf{P F}_{6}\right)_{2}\left(70 \mu \mathrm{L}, 1.910^{-4} \mathrm{M}\right)$, 3. $\left(\mathbf{P F}_{6}\right)_{2}$ $\left(70 \mu \mathrm{L}, 2.010^{-4} \mathrm{M}\right)$ and $4 .\left(\mathrm{PF}_{6}\right)_{2}\left(70 \mu \mathrm{L}, 2.210^{-4} \mathrm{M}\right)$ were added to $6 \mathrm{~mL}$ of the previous solution of Au-NPs or Ag-NPs in DCM. The different mixtures were stirred during $48 \mathrm{~h}$ at $25^{\circ} \mathrm{C}$ before analysis.

\section{Acknowledgements}

We thank Christine Longin and Sophie Chat for TEM analyses, and Julien Dubois for help in the nanosecond lifetime experiments. This work was supported by the PRES UniverSud Paris and the ANR "Jeunes Chercheuses et Jeunes Chercheurs" in the frame of AURUS program.

\section{Appendix A. Supplementary data}

Supplementary data to this article can be found online at https:// doi.org/10.1016/j.apsusc.2019.143847.

\section{References}

[1] a) U. Schatzschneider, Eur. J. Inorg. Chem. 10 (2010) 1451; b) M.J. Rose, P.K. Mascharak, Coord. Chem. Rev. 252 (2008) 2093.

[2] J.F. Endicott, Y.-J. Chen, Coord. Chem. Rev. 251 (2007) 328.

[3] a) L. Spiccia, G.B. Deacon, C.M. Kepert, Coor. Chem. Rev. 248 (2004) 1329; b) Q. Sun, S. Mosquera-Vazquez, Y. Suffren, J. Hankache, N. Amstutz, L.M. Lawson Daku, E. Vauthey, A. Hauser, Coord. Chem. Rev. 282 (2015) 87.

[4] a) S. Bonnet, J.-P. Collin, Chem. Soc. Rev. 8 (2008) 1490; b) E. Baranoff, J.P. Collin, L. Flamigni, J.-P. Sauvage, Chem. Soc. Rev. 33 (2004) 147-155;

c) P.P. Laine, S. Campagna, F. Loiseau, Coordination Chemistry Reviews 252 (2008) 2552-2571.

[5] W.K. Chan, Coord. Chem. Rev. 251 (2007) 2104

[6] O. Maury, H. Le Bozec, Acc. Chem. Soc. Res. 38 (2005) 691.

[7] L. Moriggi, A. Aebischer, C. Cannizzo, A. Sour, A. Borel, J.-C. G, L. Helm Bünzli, Dalton Trans. (12) (2009) 2088.

[8] S. Zanarini, E. Rampazzo, L. Della Ciana, M. Marcaccio, E. Marzocchi, M. Montalti, F. Paolucci, L. Prodi, J. Am. Chem. Soc. 131 (2009) 2260.

[9] J.K. Hurst, J.L. Cape, A.E. Clark, S. Das, C.Y. Qin, Inorg. Chem. 47 (2008) 1753.

[10] M.K. Nazeeruddin, S.M. Zakeeruddin, J.J. Lagref, P. Liska, P. Comte, C. Barolo, G. Viscardi, K. Schenk, M. Grätzel, Coord. Chem. Rev. 248 (2004) 1317.

[11] M.J. Li, Z.F. Chen, V.W.W. Yan, Y.B. Zu, ACS Nano 2 (2008) 905

[12] R. Kawano, M.K. Nazeerudin, A. Sato, M. Grätzel, M. Watanabe, Electrochem. Commun. 9 (2007) 1134

[13] a) C.G. Cameron, P.G. Pickup, J. Am. Chem. Soc. 121 (1999), 11773; b) M. Abe, H. Futagawa, T. Ono, T. Yamada, N. Kimizuka, Y. Hisaeda, Inorg. Chem. 54 (2015), 11061.

[14] a) T. Le Bouder, O. Maury, A. Bondon, K. Costuas, E. Amouyal, I. Ledoux, J. Zyss, H. Le Bozec, J. Am. Chem. Soc. 125 (2003), 12284;

b) F. Puntoriero, F. Nastasi, M. Cavazzini, S. Quici, S. Campagna, Coord. Chem. Rev. 251 (2007) 536;

c) J.-L. Wang, X. Li, C.D. Shreiner, X. Lu, C.N. Moorefield, S.R. Tummalapalli, D.A Medvetz, M.J. Panzner, F.R. Fronczek, C. Wesdemiotis, G.R. Newkome, New J. Chem. 36 (2012) 484

[15] a) A. Guerrero-Martinez, Y. Vida, D. Dominguez-Gutierrez, R.Q. Albuquerque, L. De Cola, Inorg. Chem. 47 (2008) 9131;

b) R. Sangiliapillai, R. Arumugam, R. Eswaran, R. Seenivasan, Luminescence 31 (2016) 30-37.

[16] a) X.D. Wu, Y. Cong, Y.H. Liu, J. Ying, B. Li, J. Sol-Gel Sci. Technol. 49 (2009) 355; b) P. Innocenzi, H. Kozuka, T. Yoko, J. Phys. Chem. B 101 (1997) 2285.

[17] a) D.L. Ma, A.J. Kell, S. Tan, Z.J. Jakubek, B. Simard, J. Phys. Chem. C 113 (2009), 15974;

b) M. Frasconi, Z. Liu, J. Lei, Y. Wu, E. Strekalova, D. Malin, M.W. Ambrogio, X Chen, Y.Y. Botros, V.L. Cryns, J.-P. Sauvage, J.F. Stoddart, J. Am. Chem. Soc. 135 (2013) 11603-11613.

[18] a) S. Verma, P. Kar, A. Das, D.K. Palit, N.H. Ghosh, Chem. Eur. J. 16 (2010) 611 b) N.-N. Zhang, F. Bigdeli, Q. Miao, M.-L. Hu, A. Morsali Journal of Organometallic Chemistry 878 (19) (2018) 11-18.
[19] See for example: a) J. Zhao, J. A. Dieringer, X. Y. Zhang, G. C. Schatz, R. P. Van Duyne J. Phys. Chem. B. 2008, 112, 19302.b G. Lemercier, M. Four, S. Chevreux, Coord. Chem. Rev. 368 (2018) 1.

[20] M.C. Daniel, D. Astruc, Chem. Rev. 104 (2004) 293.

[21] M. Brust, M. Walker, D. Bethell, D.J. Schiffrin, R. Whyman, Chem. Commun. (1994) 801.

[22] J.L. Gong, C.B. Mullins, Acc. Chem. Res. 42 (2009) 1063.

[23] a) J.H. Lee, M.A. Mahmoud, V. Sitterle, J. Sitterle, J.C. Meredith, J. Am. Chem. Soc. 131 (2009) 5048;

b) A.M. Schwartzberg, J.Z. Zhang, J. Phys. Chem. B 112 (2008), 10323.

[24] a) A. Franca, B. Pelaz, M. Moros, C. Sanchez-Espinel, A. Hernandez, C. Fernandez-Lopez, V. Grazu, J.M. de la Fuente, I. Pastoriza-Santos, L.M. Liz-Marzan, A. Gonzalez-Fernandez, Small 131 (2009) 5048;

b) P.K. Jain, X. Huang, I.H. El-Sayed, M.A. El-Sayed, Acc. Chem. Res. 41 (2008) 1578 .

[25] a) A.N. Shipway, M. Lahav, R. Blonder, I. Willner, Chem. Mater. 11 (1999) 13; b) M. Lahav, V. Heleg-Shabtai, J. Wasserman, E. Katz, I. Willner, H. Dürr, Y.-Z. Hu, S.H. Bossmann, J. Am. Chem. Soc. 122 (2000), 11480;

c) T.B. Norsten, B.L. Frankamp, V.M. Rotello, Nano Lett. 2 (2002) 1345; d) X.-H.N. Xu, S. Huang, W. Brownlow, K. Salaita, R.B. Jeffers, J. Phys. Chem. B 108 (2004) 15543;

e) P.P.H. Cheng, D. Silvester, G. Wang, G. Kalyuzhny, A. Douglas, R.W. Murray, J. Phys. Chem. B 110 (2006) 4637.

[26] a) J. F. Hicks, D. T. Miles, R. W. Murray, J. Am. Chem. Soc. 2002, 124, 13322;b) R.L. Wolfe, R. Balasubramanian, J.B. Tracy, R.W. Murray, Langmuir 23 (2007) 2247.

[27] a) B. I. Ipe, K. Yoosaf, K. G. Thomas, J. Am. Chem. Soc. 2006, 128, 1907;b) T. Huang, R.W. Murray, Langmuir 18 (2002) 7077.

[28] a) W.S. Baker, B.I. Lemon, R.M. Crooks, J. Phys. Chem. B 105 (2001) 8885 b) J.D.E.T. Wilton-Ely, Dalton Trans. 25 (2008).

[29] W.R. Glomm, S.J. Moses, M.K. Brennaman, J.M. Papanikolas, S. Franzen, J. Phys Chem. B 109 (2005) 804.

[30] a) M. Ito, T. Tsukatani, H. Fujihara, J. Mater. Chem. 15 (2005) 960; b) N. Vilvamani, M. Chhatwal, I. Bhowmick, R. Devi Gupta, S.K. Awasthi, RSC Adv. 6 (2016), 55507.

[31] C. Querner, P. Reiss, J. Bleuse, A. Pron, J. Am. Chem. Soc. 126 (2004), 11574.

[32] C. Pérez Leon, L. Kador, B. Peng, M. Thelakkat, J. Phys. Chem. B 109 (2005) 5783.

[33] a) C.R. Mayer, E. Dumas, F. Sécheresse, Chem. Commun. (2005) 345; b) C.R. Mayer, E. Dumas, A. Michel, F. Sécheresse, Chem. Commun. 40 (2006) 4183;

c) C.R. Mayer, E. Dumas, F. Sécheresse, J. Coll. Interf. Sci. 328 (2008) 452

[34] C.R. Mayer, E. Dumas, F. Miomandre, R. Méallet-Renault, F. Warmont, J. Vigneron, R. Pansu, A. Etcheberry, F. Sécheresse, New J. Chem. 30 (2006) 1628.

[35] N. Nerambourg, M.H.V. Werts, M. Charlot, M. Blanchard-Desce, Langmuir 23 (2007) 5563.

[36] C. Querner, A. Benedetto, R. Demadrille, P. Rannou, P. Reiss, Chem. Mater. 18 (2006) 4817.

[37] T.-H. Park, M.J. Therien, Org. Lett. 9 (2007) 2779.

[38] M.S. Vickers, J. Cookson, P.D. Beer, P.T. Bishop, B. Thiebaut, J. Mater. Chem. 16 (2006) 209

[39] J.E. Collins, J.J.S. Lamba, J.C. Love, J.E. McAlvin, C. Ng, B.P. Peters, X. Wu, C.L. Fraser, Inorg. Chem. 38 (1999) 2020.

[40] S.R. Ramadas, P.S. Srinivasan, J. Ramachandran, V.V.S.K. Sastry, Synthesis (1983) 605.

[41] S. Rucareanu, V.J. Gandubert, R.B. Lennox, Chem. Mater. 18 (2006) 4674

[42] J.D.S. Newman, G.J. Blanchard, Langmuir 22 (2006) 5882

[43] P. Pramod, P.K. Sudeep, K.G. Thomas, P.V. Kamat, J. Phys. Chem. B 110 (2006), 20737.

[44] I. Ojea-Jiménez, V. Puntes, J. Am. Chem. Soc. 131 (2009), 13320.

[45] a) L.L. Rouhana, J.A. Jaber, J.B. Schlenoff, Langmuir 23 (2007), 12799; b) S. Tan, M. Erol, S. Sukhishvili, H. Du, Langmuir 24 (2008) 4765.

[46] M. Jebb, P.K. Sudeep, P. Pramod, K.G. Thomas, P.V. Kamat, J. Phys. Chem. B 111 (2007) 6839

[47] a) A. Kumar, S. Mandal, P.R. Selvakannan, R. Pasricha, A.B. Mandale, M. Sastry, Langmuir 19 (2003) 6277;

b) M. Yamamoto, Y. Kashiwagi, M. Nakamoto, Langmuir 22 (2006) 8581.

[48] a) H. Chao, R.-H. Li, C.-W. Jiang, H. Li, L.-N. Ji, X.-Y. Ji, Dalton Trans 2001 (1920);

b) M. Mariappan, B.G. Maiya, Eur. J. Inorg. Chem. (2005) 2164

[49] See for example: L.-F. Tan, F. Wang, H. Chao, Hel. Chim. Acta 2007, 90, 205 and references therein.

[50] K. Nakamaru, Bull. Chem. Soc. Jpn. 55 (1982) 2697.

[51] a) K.-C. Lee, S.-J. Lin, C.-H. Lin, C.-S. Tsai, Y.-J. Lu, Surf. Coat. Techn. 202 (2008) 5339;

b) W. Wang, X. Chen, S. Efrima, J. Phys. Chem. B 103 (1999) 7238; c) E. Oh, K. Susumu, R. Goswami, H. Mattoussi, Langmuir ASAP (2010).

[52] J.V. Caspar, T.J. Meyer, J. Am. Chem. Soc. 105 (1983) 5583.

[53] B. Durham, J.V. Caspar, J.K. Nagle, T.J. Meyer, J. Am. Chem. Soc. 104 (1982) 4803.

[54] a) T.J. Meyer, Pure Appl. Chem. 58 (1986) 1193; b) A. Masschelein, L. Jacquet, A. Kirsch-De Mesmaeker, J. Nasielski, Inorg. Chem. 29 (1990) 855.

[55] H. Ishida, S. Tobita, Y. Hasegawa, R. Katoh, K. Nozaki, Coord. Chem. Rev. 254 (2010) 2449. 
[56] N.H. Damrauer, T.R. Boussie, M. Devenney, J.K. McCusker, J. Am. Chem. Soc. 119 (1997) 8253.

[57] K.G. Thomas, P.V. Kamat, Acc. Chem. Res. 36 (2003) 888.

[58] E. Dulkeith, A.C. Morteani, T. Niedereichholz, T.A. Klar, J. Feldmann, S.A. Levi, F.C.J.M. van Veggel, D.N. Reinhoudt, M. Möller, D. I. Gittins 203002 (2002) 89.

[59] J.R. Lakowicz, Principles of Fluorescence Spectroscopy, Second edition, Kluwer Academic/Plenum Press, New York, 1999.

[60] A.J. Bard, L.R. Faulkner, Electrochemical Methods: Fundamentals and Applications, $2^{\text {nd }}$ ed., Wiley, NewYork, 2001.

[61] R.W. Murray, Chem. Rev. 108 (2008) 2688.

[62] N.K. Chaki, B. Kakade, K.P. Vijayamohanan, P. Singh, C.V. Dharmadhikari, Phys. Chem. Chem. Phys. 8 (2006) 1837.

[63] S.J. Green, J.J. Stokes, M.J. Hostetler, J. Pietron, R.W. Murray, J. Phys. Chem. B 101 (1997) 2663.

[64] Estimated from ref., E. W. Constable, E. Figgemeier, C. H. Housecroft, J. Olsson, Y. C, Zimmermann, Dalton Trans, 20041918
[65] C. Hiort, P. Lincoln, B. Nordén, J. Am. Chem. Soc. 115 (1993) 3448

[66] C.A. Goss, H.D. Abruna, Inorg. Chem. 24 (1985) 4263

[67] B.P. Sullivan, D.J. Salmon, T.J. Meyer, Inorg. Chem. 17 (1978) 3334.

[68] Y.-J. Liu, C.-H. Zeng, H.-L. Huang, L.-X. He, F.-H. Wu, Eur. J. Med. Chem. 45 (2010) 564

[69] L. Schoutteten, P. Denjean, R.B. Pansu, J. Fluoresc. 7 (1997) 155.

[70] Y. Pellegrin, M. Sandroni, E. Blart, A. Planchat, M. Evain, N.C. Bera, M. Kayanuma, M. Sliwa, M. Rebarz, O. Poizat, C. Daniel, F. Odobel, Inorg. Chem. 50 (2011) 11309 .

[71] D. Garreau, J.M. Savéant, J. Electroanal. Chem. 50 (1975) 1.

[72] F. Dumur, C.R. Mayer, K. Hoang-Thi, I. Ledoux-Rak, F. Miomandre, G. Clavier, E. Dumas, R. Méallet-Renault, M. Frigoli, J. Zyss, F. Sécheresse, Inorg. Chem. 48 (2009) 8120.

[73] T. Gu, J.K. Whitesell, M.A. Fox, Chem. Mater. 15 (2003) 1358. 